\title{
Search for Physics beyond the Standard Model in Events with Overlapping Photons and Jets
}

\author{
A. M. Sirunyan et al. \\ (CMS Collaboration)
}

(Received 14 July 2019; revised manuscript received 23 October 2019; published 11 December 2019)

\begin{abstract}
Results are reported from a search for new particles that decay into a photon and two gluons, in events with jets. Novel jet substructure techniques are developed that allow photons to be identified in an environment densely populated with hadrons. The analyzed proton-proton collision data were collected by the CMS experiment at the LHC, in 2016 at $\sqrt{s}=13 \mathrm{TeV}$, and correspond to an integrated luminosity of $35.9 \mathrm{fb}^{-1}$. The spectra of total transverse hadronic energy of candidate events are examined for deviations from the standard model predictions. No statistically significant excess is observed over the expected background. The first cross section limits on new physics processes resulting in such events are set. The results are interpreted as upper limits on the rate of gluino pair production, utilizing a simplified stealth supersymmetry model. The excluded gluino masses extend up to $1.7 \mathrm{TeV}$, for a neutralino mass of $200 \mathrm{GeV}$ and exceed previous mass constraints set by analyses targeting events with isolated photons.
\end{abstract}

DOI: 10.1103/PhysRevLett.123.241801

Despite the success of the standard model (SM) of particle physics, there are a number of indications, such as the cosmological observations of dark matter and the low measured value of the Higgs boson mass, that suggest the existence of new physics at the TeV energy scale. No evidence for new physics has been uncovered thus far by the LHC. Signs of new phenomena could be hidden by high rate background SM processes that have yet to be properly explored. A large number of well-motivated theoretical scenarios predict the appearance of new physics in protonproton collision events with low missing transverse momentum $\left(p_{\mathrm{T}}^{\text {miss }}\right)$ and nonisolated photons and leptons, which would appear as multijet events in a collider detector. These scenarios arise in hidden valley models [1,2] and a number of supersymmetric (SUSY) models, such as $R$ parity violating SUSY [3] and stealth SUSY [4-6].

Stealth SUSY predicts a hidden sector of particles with minimal couplings to the SUSY breaking mechanism. As a result, the superpartners in this sector are nearly mass degenerate. In the present analysis, a simplified stealth SUSY model is used as a benchmark. The model has only one light hidden sector superparticle pair, the singlino, and the singlet ( $\tilde{S}$ and $S$, respectively). Gluinos $(\tilde{g})$, the gluon superpartners, are expected to be created with large cross sections at the LHC and to decay to neutralinos $\tilde{\chi}_{1}^{0}$ and a

\footnotetext{
*Full author list given at the end of the Letter.

Published by the American Physical Society under the terms of the Creative Commons Attribution 4.0 International license. Further distribution of this work must maintain attribution to the author(s) and the published article's title, journal citation, and DOI. Funded by SCOAP ${ }^{3}$.
}

quark-antiquark pair. Stealth SUSY assumes gauginos (either neutralinos or charginos), which decay to a $\tilde{S}$ and a photon $(\gamma)$, to be the portal to the hidden sector. The $\tilde{S}$ is expected to decay to an $S$ and a massless gravitino $(\tilde{G})$, with the subsequent decay of the $S$ to a pair of gluons. Because of the mass degeneracy of the hidden-sector pair, the $\tilde{G}$ is expected to be produced with low momentum and the event to be characterized by low $p_{\mathrm{T}}^{\text {miss }}$. A diagram depicting the decay chain of a gluino according to this simplified stealth SUSY model is presented in Fig. 1.

Previous searches at CMS for stealth SUSY $[7,8]$ required two isolated photons. The isolation requirement reduces the sensitivity for scenarios where a large mass difference exists between the electroweak gauginos, in this case the $\tilde{\chi}_{1}^{0}$ and the colored superparticle $(\tilde{g})$. If this large mass interval is present, the $\tilde{\chi}_{1}^{0}$ is expected to be produced with a large Lorentz boost and its decay products to be collimated, resulting in photons that are not isolated in the event. Since we search for events with jets composed of one photon from the $\tilde{\chi}_{1}^{0}$ decay and a pair of gluons from the $S$ decay, which we refer to as photon jets, our search is complementary to previous searches. It is possible to identify photon jets by utilizing a combination of existing and novel jet substructure tools. Within the simplified stealth SUSY model we consider, superparticles would be produced at the LHC in events with two photon jets associated with a large number of hadrons. The distribution of the total transverse hadronic energy of events containing photon jets is used to discriminate possible new physics obscured by the SM multijet background.

The central feature of the CMS apparatus is a superconducting solenoid of $6 \mathrm{~m}$ internal diameter, providing a magnetic field of $3.8 \mathrm{~T}$. Within the solenoid volume are a 


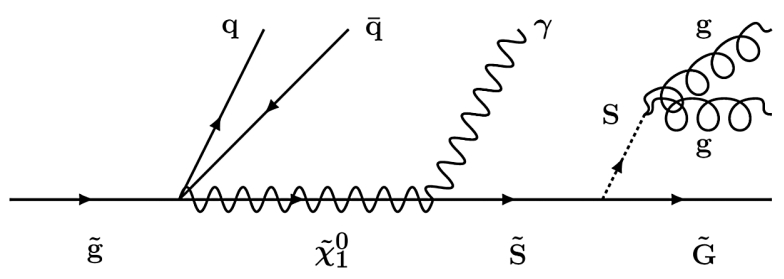

FIG. 1. The decay diagram for a single gluino as predicted by stealth SUSY. This analysis searches for pair produced gluinos and thus two such decay chains are expected in each signal event.

silicon pixel and strip tracker, a lead tungstate crystal electromagnetic calorimeter (ECAL), and a brass and scintillator hadron calorimeter, each composed of a barrel and two end cap sections. Forward calorimeters extend the pseudorapidity $(\eta)$ coverage provided by the barrel and end cap detectors. Muons are detected in gas-ionization chambers embedded in the steel flux-return yoke outside the solenoid. Observed events that are considered potentially interesting are selected by a two-tiered trigger system [9]. A more detailed description of the CMS detector, together with a definition of the coordinate system used and the relevant kinematic variables, can be found in Ref. [10].

Particle objects are reconstructed by the particle-flow algorithm [11], from combinations of observations from the CMS detector components. The particle objects are clustered into jets using the anti- $k_{\mathrm{T}}$ algorithm [12] implemented in FASTJET [13] with a distance parameter of 0.8 (AK8 jets) and 0.4 (AK4 jets). The AK4 jet collection is utilized mainly for triggering purposes, while the larger radius AK8 jet collection, for the reconstruction of the $\tilde{\chi}_{1}^{0}$ decays. The primary vertex is defined as the reconstructed vertex with the largest quadratic sum of the transverse momenta $\left(p_{\mathrm{T}}\right)$ of AK4 jets clustered from tracks associated with the vertex and the negative vector- $p_{\mathrm{T}}$ sum of these jets. Chargedparticle candidates not associated with the primary vertex are ignored to reduce pileup effects in the event reconstruction. Pileup refers to additional proton-proton $(p p)$ collisions within the same or neighboring bunch crossings of the LHC beams. Jets are required to pass loose identification criteria [14], to reduce misreconstructed jets and jets reconstructed from calorimeter noise [15]. In addition, energy corrections are applied to the jets [16]. Kinematic requirements of a minimum jet $p_{\mathrm{T}}$ of $200 \mathrm{GeV}$ and the jet pseudorapidity $(\eta)$, to be $-2<\eta<2$ are applied to AK8 jets. The AK8 jet $p_{\mathrm{T}}$ is used to measure the total transverse hadronic activity in the event, defined as $H_{\mathrm{T}}=\sum p_{\mathrm{T}}$, where the sum is over all the AK8 jets in the event. For the analysis, we consider events that have $H_{\mathrm{T}}>1 \mathrm{TeV}$ and contain at least $3 \mathrm{AK} 8$ jets.

The data analyzed were collected by the CMS experiment at the LHC from $p p$ collisions at $\sqrt{s}=13 \mathrm{TeV}$ during the 2016 data taking period, and correspond to an integrated luminosity of $35.9 \mathrm{fb}^{-1}$. Events are selected by the trigger system if they pass a minimum $H_{\mathrm{T}}$ requirement of $900 \mathrm{GeV}$, calculated using the AK4 jets with a minimum
$p_{\mathrm{T}}$ of $50 \mathrm{GeV}$ and $|\eta|<2.5$. For the purpose of correcting data-to-simulation differences, events were also collected with a combination of muon triggers, selecting events containing at least one muon with $p_{\mathrm{T}}$ greater than $50 \mathrm{GeV}$.

Pair production of gluinos for a range of different $\tilde{g}$ and $\tilde{\chi}_{1}^{0}$ masses, with the $S$ and $\tilde{S}$ masses fixed to 90 and $100 \mathrm{GeV}$, respectively, are simulated using MADGRAPH5_aMC@NLO [17]. The decay and hadronization are done with PYTHIA [18] using the CUETP8M1 tune [19] for the underlying event and the NNPDF3.0 parton distribution functions (PDF) [20]. The detector is simulated with the CMS fast simulation package (FASTSIM) [21,22]. To estimate systematic uncertainties related to the detector simulation, the full CMS detector simulation (FULLSIM) based on GEANT4 [23] is also used and its results are compared to those of FASTSIM. An uncertainty due to the hadronization model is evaluated by an alternative signal simulation with HERWIG [24] and the TUNEEE5C [25] underlying event tune. Signal events are normalized using the theoretical gluino pair production cross sections [26] at next-to-leading order, assuming a 100\% branching fraction to the $\tilde{g}$ decay channel shown in Fig. 1.

We simulate SM processes to study the behavior of the background, to construct templates from which we estimate the efficiency corrections used for simulated signals, and to estimate the various uncertainties. The dominant background is from quantum chromodynamic (QCD) multijet processes. Simulation of QCD processes is done using MADGRAPH5AMC@NLO with MLM matching [27] and hadronized with PYTHIA8 with the CUETP8M1 tune. The production of hadronically and leptonically decaying $W$ bosons in conjunction with jets ( $W+$ jets) is also simulated this way. Top quark-antiquark pairs $(t \bar{t})$ are simulated with POWHEGV2 [28-31] and hadronized by PYTHIA8 using the CUETP8M2T4 [19] underlying event tune. As an alternative to PYTHIA, HERWIG with the TUNEEE5C underlying event tune are also used for hadronization of $t \bar{t}$ pairs. All samples are simulated with the NNPDF3.0 PDFs. The detector response is simulated using GEANT4.

Each AK8 jet in the event is examined to identify candidate photon jets, which will have a three-prong substructure and a photon from the $\tilde{\chi}_{1}^{0}$ decay. We require that there is at least one photon cluster in the AK8 jet, with $p_{\mathrm{T}}>20 \mathrm{GeV}$ and at least $95 \%$ of the energy deposited in ECAL, consistent with a photon shower shape [32]. This photon candidate is also required to not have any associated hits in the pixel detector (pixel veto). Photons converting in the tracker material can produce multiple PF objects, which are replaced by the reconstructed photon object four vector. The photon and the AK8 jet constituents are reclustered using the $k_{\mathrm{T}}$ algorithm [33] and the merging history is examined to identify the three subjets of the jet. The clustering algorithm combines two objects into one at each step. We identify as the first subjet, the less massive of the two objects merged in the last step of the clustering sequence. The other object, the more massive of the two, specifies the second and third subjets. To be considered a photon jet, the AK8 jet must have three 


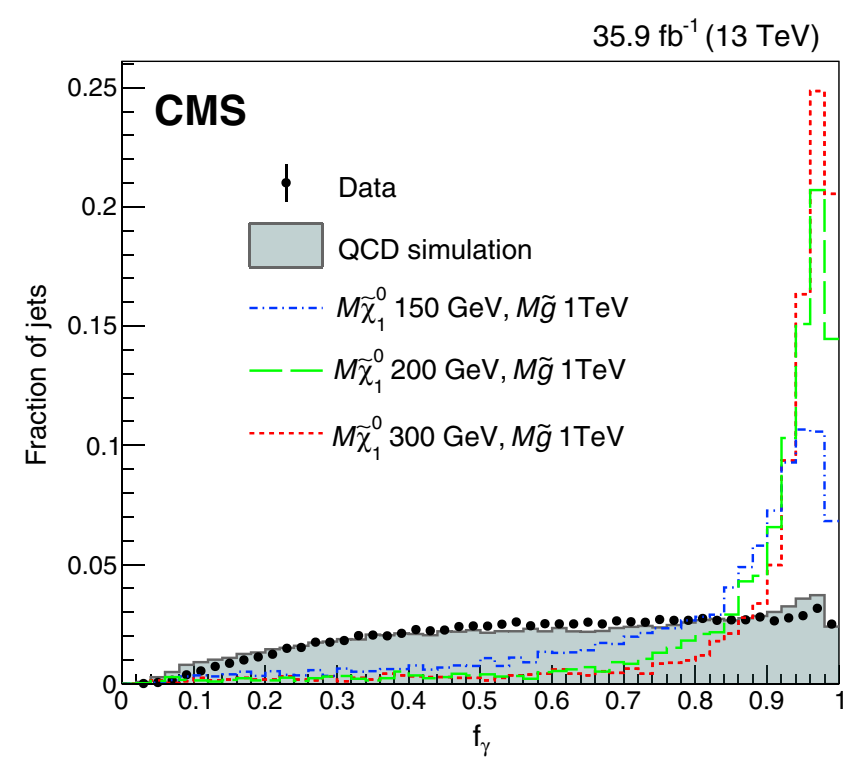

FIG. 2. Distribution of the photon subjet energy fraction $\left(f_{\gamma}\right)$ for jets that satisfy the loose photon jet requirements. Simulated distributions for signal are denoted by the broken lines, each depicting a different mass of $\tilde{\chi}_{1}^{0}$ and $\tilde{g}$. The shaded area represents the QCD jets distribution.

subjets with $p_{\mathrm{T}}>10 \mathrm{GeV}$. We further examine the subjet that contains the photon and define the photon subjet energy fraction $\left(f_{\gamma}\right)$ as the ratio of the photon's transverse energy to the subjet's $p_{\mathrm{T}}$. The $f_{\gamma}$ distribution is shown in Fig. 2 for data, simulated multijet backgrounds, and simulated signal. This variable is a measure of the activity around the photon and serves as a strong discriminator against the QCD multijet background.

An additional jet-substructure tool is used to enhance the discrimination between signal like three-prong jets, and background dominated single prong jets. In this approach, the $N$-subjettiness variables [34] denoted by $\tau_{N}$ are used to determine the consistency of a jet with $N$ or fewer prongs. The $\tau_{N}$ values are defined as the following:

$$
\tau_{N} \equiv \frac{1}{d_{0}} \sum_{i} p_{\mathrm{T} i} \min \left\{\Delta R_{1, i}, \Delta R_{2, i}, \ldots \Delta R_{N, i}\right\},
$$

where the index $i$ refers to each jet constituent, $\Delta R$ is the angular distance between a jet constituent and a candidate subjet axis, and $d_{0}$ is a normalization constant. Jets composed of three subjets should have small values for the ratio $\tau_{3} / \tau_{1}$. Photon jets are required to satisfy the condition $\tau_{3} / \tau_{1}<0.4$. Photon jets satisfying the additional requirement $f_{\gamma}>0.9$ are categorized as tight photon jets, the rest are referred to as loose photon jets. Events are characterized by their multiplicity of loose and tight photon jets, and are labeled as $X-Y$ where $X$ is the number of loose photon jets, of which $Y$ also satisfies the tight photon jet criteria. We define the signal region (SR) as that containing events with exactly two loose photon jets, while the background dominated region (BR) contains events with one or less loose photon jet. The SR is further split into three multiplicity categories, 2-0, 2-1, and 2-2, with the last one being the most sensitive to the signal.

The SM multijet background is estimated from data. The probabilities for a QCD jet to be labeled as a loose or tight photon jet, referred to as mistag rates, are measured in the BR as a function of the jet $p_{\mathrm{T}}$ and $\eta$. The loose mistag rate is measured by taking the ratio of the number of jets passing the loose selection in the BR, to the total number of the jets in the $\mathrm{BR}$, as a function of jet $p_{\mathrm{T}}$ and $\eta$. The tight photon jet mistag rate is the ratio of the number of tight photon jets to the number of all loose photon jets in the BR. The probabilities of each event to populate the three SR categories are calculated by generating an ensemble of $10^{4}$ pseudoexperiments for each event in the BR, using the AK8 jet kinematic variables and the measured mistag rates. One can then obtain the background $H_{\mathrm{T}}$ distributions, for each SR category. This is achieved by constructing an $H_{\mathrm{T}}$ distribution of all events in the BR and weighting each event by the calculated probabilities for it to pass the SR selections. The mistag rates are varied within their statistical uncertainties to determine the uncertainty in the background prediction. It was found that the background contribution is underestimated in events where overlap between neighboring jets exists. Therefore, in each event, the minimum pairwise distance in the $\eta-\phi$ space between AK8 jets, defined as $\Delta R=\sqrt{(\Delta \eta)^{2}+(\Delta \phi)^{2}}$, is required to be $\Delta R>1.5$. The validity of the background method is tested by confirming that there is agreement between prediction and observation for the numbers of events in the $H_{\mathrm{T}}$ distributions and for their shapes. The tests are performed both with simulated events and with a subset of the data corresponding to $10 \%$ of the total integrated luminosity. In each case the method is found to achieve closure to within 5\%. Other SM processes such as $t \bar{t}$ and $W+$ jets are simulated and estimated to have a negligible contribution in the SR.

To measure the signal efficiency correction for the loose and tight photon jet selections, since no SM process predicts jets composed of a collimated photon and two gluons, we select AK8 jets that are composed of an electron, a bottom quark and a final-state radiation gluon, originating from top quark decays. This approach requires the pixel veto constraint to be reversed in order to allow an electron in a jet to emulate a photon. A $t \bar{t}$ dominated sample is selected by tagging events in which the combination of a muon, a loosely $b$-tagged AK4 jet [35] and $p_{\mathrm{T}}^{\text {miss }}$ is back to back to an AK8 jet (probe jet). The probe jets are used for the measurement of the loose and tight photon jet rates. The measurement is done by fitting simulation-based templates to the probe jets, estimating the data composition (e.g., jets originating from light quarks or gluons, or fully merged hadronic $\mathrm{W}$ boson or top quark decays) and measuring the loose and tight photon jets selection efficiency. The procedure is repeated in simulation and the efficiency correction is defined as the ratio of the loose or tight efficiency measured in data over the one obtained from $t \bar{t}$ simulation. The templates are constructed using the probe 
TABLE I. Impact of systematic uncertainties on either signal acceptance $\left({ }^{s}\right)$ or background $\left({ }^{b}\right)$. Shape uncertainties are denoted by an asterisk $\left(^{*}\right)$, while the others are considered normalization uncertainties.

\begin{tabular}{lr}
\hline \hline Source & Impact \\
\hline Simulation-to-data signal efficiency correction ${ }^{* s}$ & $30-50 \%$ \\
Background estimation $^{* b}$ & $5-20 \%$ \\
Jet energy resolution $^{* s b}$ & $<10 \%$ \\
Jet energy scale corrections $^{* s b}$ & $<10 \%$ \\
Pileup reweighting $^{* s}$ & $<5 \%$ \\
Integrated luminosity $^{s}$ & $2.5 \%$ \\
Detector FULLSIM-FASTSIM $^{s}$ & $1-2 \%$ \\
PDF choice uncertainty $^{s}$ & $1 \%$ \\
\hline \hline
\end{tabular}

jet mass. Using simulated top pairs and signal samples hadronized with PYTHIA and HERWIG, an uncertainty is derived to address the differences in the jet constituents between top and signal jets. Finally, the signal yield is scaled to correct for the difference between data and simulation, and the associated uncertainty in the yield is estimated by measuring the impact of changing the scaling factor by its uncertainty.
The dominant source of systematic uncertainty is the data-to-simulation efficiency correction for signal-like jets. This ranges from 30 to $50 \%$ depending on the event jet composition. The uncertainties considered and their magnitudes are listed in Table I. These include uncertainties associated with the following sources: background estimation, jet calibration and resolution corrections, which can affect the measured jet energy [36], pileup modeling, the total integrated luminosity measurement [37], simulation effects for signal such as the difference between the full and fast detector simulation, and the PDF choice [38]. Initialstate radiation effects on signal efficiency and triggering efficiency uncertainties are estimated to be negligible and not included. Systematic uncertainties are introduced as shape or normalization variations for the limit setting procedure, as indicated in Table I.

The search is performed separately on events with exactly three AK8 jets and events with four or more AK8 jets. A joint statistical analysis is performed using the $H_{\mathrm{T}}$ spectra in the six SR considered. The $H_{\mathrm{T}}$ distributions in the SR are presented in Fig. 3, where it can be seen that the data are consistent with the background prediction. We interpret the results as upper limits on the cross section for pair-produced
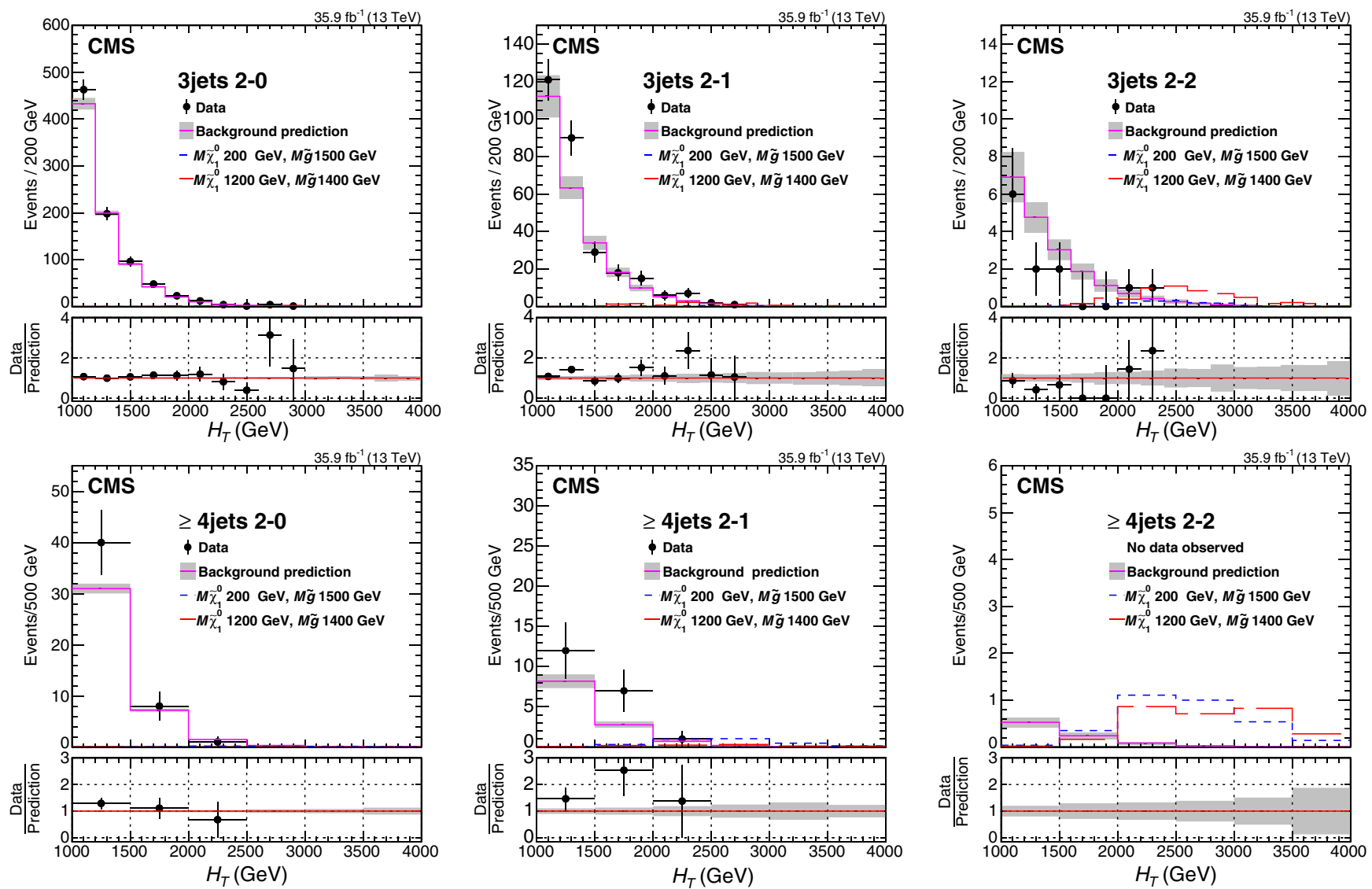

FIG. 3. The $H_{\mathrm{T}}$ distributions in the signal regions for the three-jet AK8 (upper row) and the $\geq 4$ AK8 jets categories (lower row). Events with zero, one, and two tight photon jets are presented from left to right. The magenta line with the gray band corresponds to the background expectation obtained from data while the blue and red colored lines present two signal benchmarks. The lower panels present the data-to-background ratio with their respective uncertainties. 


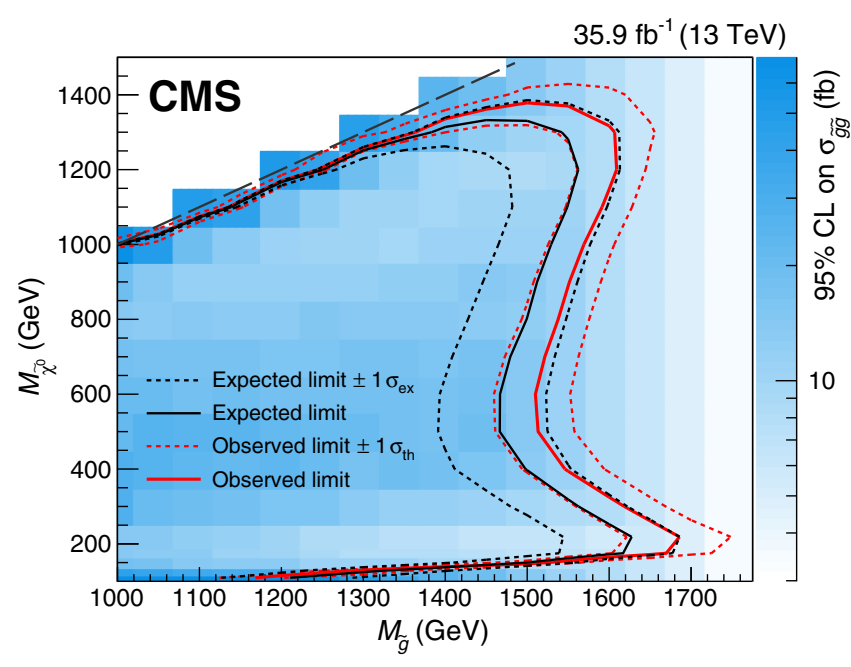

FIG. 4. The upper limit at $95 \%$ confidence level on the $\tilde{g}$ pair production cross section as a function of $\tilde{g}$ and $\tilde{\chi}_{1}^{0}$ masses. The region enclosed by the red (lighter) solid line is excluded. The black (darker) solid line presents the expected excluded area. The uncertainty in the observed limit corresponds to the theoretical uncertainties in the signal cross section. Exclusion in the low $\tilde{\chi}_{1}^{0}$ and high $\tilde{g}$ mass region is a result of the implementation of the substructure techniques.

gluinos, decaying according to the simplified stealth SUSY model, using a Bayesian limit setting method with a flat signal prior [39]. The systematic uncertainties are incorporated as nuisance parameters with log-normal priors and are assumed to be correlated among the six SR. The cross section limits for all SR categories are shown in Fig. 4. Production of $\tilde{g}$ with masses up to $1.7 \mathrm{TeV}$ are excluded at a $95 \%$ confidence level, for an assumed $\tilde{\chi}_{1}^{0}$ mass of $200 \mathrm{GeV}$. For neutralino masses between 1.0 and $1.2 \mathrm{TeV}$, the maximum excluded gluino mass is $1.5-1.7 \mathrm{TeV}$. This is the first result on boosted final states with photons and gluons merging into a single jet. The resulting limits improve over those obtained in previous analyses searching for isolated photons.

To summarize, a search for new particles decaying to a photon and two gluons in events with jets is presented. The search is performed in events with two jets that have substructure and are composed of a photon and two gluons. A dataset of proton-proton collisions at a center-of-mass energy of $13 \mathrm{TeV}$ collected by the CMS experiment, corresponding to an integrated luminosity of $35.9 \mathrm{fb}^{-1}$, is analyzed. To identify the candidate jets, novel jet substructure techniques have been developed and used to complement established methods. The total transverse hadronic activity distributions of events in the signal region are compared to the expected distributions, estimated from data. No statistically significant excess is observed above the standard model background expectation. We establish upper limits at $95 \%$ confidence level on the cross section for gluino pair production, using a simplified stealth SUSY model. The excluded gluino masses extend up to
1.5-1.7 TeV, depending on the neutralino mass, with the highest exclusion set for neutralinos with a mass of $200 \mathrm{GeV}$. This is the first search of this kind targeting the region of parameter space where photons from neutralino decays are not isolated.

We congratulate our colleagues in the CERN accelerator departments for the excellent performance of the LHC and thank the technical and administrative staffs at CERN and at other CMS institutes for their contributions to the success of the CMS effort. In addition, we gratefully acknowledge the computing centers and personnel of the Worldwide LHC Computing Grid for delivering so effectively the computing infrastructure essential to our analyses. Finally, we acknowledge the enduring support for the construction and operation of the LHC and the CMS detector provided by the following funding agencies: BMBWF and FWF (Austria); FNRS and FWO (Belgium); CNPq, CAPES, FAPERJ, FAPERGS, and FAPESP (Brazil); MES (Bulgaria); CERN; CAS, MoST, and NSFC (China); COLCIENCIAS (Colombia); MSES and CSF (Croatia); RPF (Cyprus); SENESCYT (Ecuador); MoER, ERC IUT, PUT and ERDF (Estonia); Academy of Finland, MEC, and HIP (Finland); CEA and CNRS/IN2P3 (France); BMBF, DFG, and HGF (Germany); GSRT (Greece); NKFIA (Hungary); DAE and DST (India); IPM (Iran); SFI (Ireland); INFN (Italy); MSIP and NRF (Republic of Korea); MES (Latvia); LAS (Lithuania); MOE and UM (Malaysia); BUAP, CINVESTAV, CONACYT, LNS, SEP, and UASLP-FAI (Mexico); MOS (Montenegro); MBIE (New Zealand); PAEC (Pakistan); MSHE and NSC (Poland); FCT (Portugal); JINR (Dubna); MON, RosAtom, RAS, RFBR, and NRC KI (Russia); MESTD (Serbia); SEIDI, CPAN, PCTI, and FEDER (Spain); MOSTR (Sri Lanka); Swiss Funding Agencies (Switzerland); MST (Taipei); ThEPCenter, IPST, STAR, and NSTDA (Thailand); TUBITAK and TAEK (Turkey); NASU and SFFR (Ukraine); STFC (United Kingdom); DOE and NSF (USA).

[1] M. J. Strassler and K. M. Zurek, Echoes of a hidden valley at hadron colliders, Phys. Lett. B 651, 374 (2007).

[2] M. J. Strassler, Possible effects of a hidden valley on supersymmetric phenomenology, arXiv:hep-ph/0607160.

[3] R. Barbier, C. Bérat, M. Besançon, M. Chemtob, A. Deandrea, E. Dudas, P. Fayet, S. Lavignac, G. Moreau, E. Perez, and Y. Sirois, R-parity-violating supersymmetry, Phys. Rep. 420, 1 (2005).

[4] J. Fan, M. Reece, and J. T. Ruderman, A stealth supersymmetry sampler, J. High Energy Phys. 07 (2012) 196.

[5] J. Fan, M. Reece, and J. T. Ruderman, Stealth supersymmetry, J. High Energy Phys. 11 (2011) 012.

[6] J. Fan, R. Krall, D. Pinner, M. Reece, and J. T. Ruderman, Stealth supersymmetry simplified, J. High Energy Phys. 07 (2016) 016. 
[7] CMS Collaboration, Search for stealth supersymmetry in events with jets, either photons or leptons, and low missing transverse momentum in pp collisions at $8 \mathrm{TeV}$, Phys. Lett. B 743, 503 (2015).

[8] CMS Collaboration, Search for supersymmetry in events with photons and low missing transverse energy in pp collisions at $\sqrt{s}=7 \mathrm{TeV}$, Phys. Lett. B 719, 42 (2013).

[9] CMS Collaboration, The CMS trigger system, J. Instrum. 12, P01020 (2017).

[10] CMS Collaboration, The CMS experiment at the CERN LHC, J. Instrum. 3, S08004 (2008).

[11] CMS Collaboration, Particle-flow reconstruction and global event description with the CMS detector, J. Instrum. 12, P10003 (2017).

[12] M. Cacciari, G. P. Salam, and G. Soyez, The anti- $k_{\mathrm{T}}$ jet clustering algorithm, J. High Energy Phys. 04 (2008) 063.

[13] M. Cacciari, G. P. Salam, and G. Soyez, FastJet user manual, Eur. Phys. J. C 72, 1896 (2012).

[14] CMS Collaboration, Jet algorithms performance in $13 \mathrm{TeV}$ data, CMS physics analysis summary CMS-PAS-JME-16003, 2016, https://cds.cern.ch/record/2256875.

[15] CMS Collaboration, Identification and filtering of uncharacteristic noise in the CMS hadron calorimeter, J. Instrum. 5, T03014 (2010).

[16] CMS Collaboration, Jet energy scale and resolution in the CMS experiment in pp collisions at $8 \mathrm{TeV}$, J. Instrum. 12, P02014 (2017).

[17] J. Alwall, R. Frederix, S. Frixione, V. Hirschi, F. Maltoni, O. Mattelaer, H. S. Shao, T. Stelzer, P. Torrielli, and M. Zaro, The automated computation of tree-level and next-toleading order differential cross sections, and their matching to parton shower simulations, J. High Energy Phys. 07 (2014) 079.

[18] T. Sjöstrand, S. Ask, J. R. Christiansen, R. Corke, N. Desai, P. Ilten, S. Mrenna, S. Prestel, C. O. Rasmussen, and P. Z. Skands, An introduction to PYTHIA 8.2, Comput. Phys. Commun. 191, 159 (2015).

[19] CMS Collaboration, Event generator tunes obtained from underlying event and multiparton scattering measurements, Eur. Phys. J. C 76, 155 (2016).

[20] R. D. Ball, V. Bertone, S. Carrazza, C. S. Deans, L. Del Debbio, S. Forte, A. Guffanti, N. P. Hartland, J. I. Latorre, J. Rojo, and M. Ubiali (NNPDF Collaboration), Parton distributions with LHC data, Nucl. Phys. B867, 244 (2013).

[21] S. Abdullin, P. Azzi, F. Beaudette, P. Janot, and A. Perrotta, The fast simulation of the CMS detector at LHC, Proceedings, 18th International Conference on Computing in High Energy and Nuclear Physics (CHEP 2010), (IOP Publishing, Taipei, Taiwan, 2010); J. Phys. Conf. Ser. 331, 032049 (2011).

[22] A. Giammanco, The fast Simulation of the CMS Experiment, Proceedings, 20th International Conference on Computing in High Energy and Nuclear Physics (CHEP 2013): Amsterdam, The Netherlands, 2013 (IOP Publishing, Bristol, 2013); The Fast Simulation of the CMS Experiment, J. Phys. Conf. Ser. 513, 022012 (2014).
[23] S. Agostinelli et al. (GEANT4 Collaboration), GEANT4-A simulation toolkit, Nucl. Instrum. Methods Phys. Res., Sect. A 506, 250 (2003).

[24] M. Bähr, S. Gieseke, M. A. Gigg, D. Grellscheid, K. Hamilton, O. Latunde-Dada, S. Plätzer, P. Richardson, M. H. Seymour, A. Sherstnev, and B. R. Webber, Herwig + + physics and manual, Eur. Phys. J. C 58, 639 (2008).

[25] S. Gieseke, C. Rohr, and A. Siodmok, Colour reconnections in Herwig + +, Eur. Phys. J. C 72, 2225 (2012).

[26] C. Borschensky, M. Krämer, A. Kulesza, M. Mangano, S. Padhi, T. Plehn, and X. Portell, Squark and gluino production cross sections in pp collisions at $\sqrt{s}=13,14,33$ and 100 TeV, Eur. Phys. J. C 74, 3174 (2014).

[27] J. Alwall, S. Höche, F. Krauss, N. Lavesson, L. Lönnblad, F. Maltoni, M. L. Mangano, M. Moretti, C. G. Papadopoulos, F. Piccinini, S. Schumann, M. Treccani, J. Winter, and M. Worek, Comparative study of various algorithms for the merging of parton showers and matrix elements in hadronic collisions, Eur. Phys. J. C 53, 473 (2008).

[28] P. Nason, A new method for combining NLO QCD with shower Monte Carlo algorithms, J. High Energy Phys. 11 (2004) 040.

[29] S. Frixione, P. Nason, and C. Oleari, Matching NLO QCD computations with parton shower simulations: the POWHEG method, J. High Energy Phys. 11 (2007) 070.

[30] S. Alioli, P. Nason, C. Oleari, and E. Re, A general framework for implementing NLO calculations in shower Monte Carlo programs: the POWHEG BOX, J. High Energy Phys. 06 (2010) 043.

[31] S. Frixione, P. Nason, and G. Ridolfi, A positive-weight next-to-leading-order Monte Carlo for heavy flavour hadroproduction, J. High Energy Phys. 09 (2007) 126.

[32] CMS Collaboration, Performance of photon reconstruction and identification with the CMS detector in proton-proton collisions at $\operatorname{sqrt}(\mathrm{s})=8 \mathrm{TeV}, \mathrm{J}$. Instrum. 10, P08010 (2015).

[33] S. Catani, Y. L. Dokshitzer, M. H. Seymour, and B. R. Webber, Longitudinally invariant $k_{\mathrm{T}}$ clustering algorithms for hadron hadron collisions, Nucl. Phys. B406, 187 (1993).

[34] J. Thaler and K. Van Tilburg, Identifying boosted objects with $N$-subjettiness, J. High Energy Phys. 03 (2011) 15.

[35] CMS Collaboration, Identification of heavy-flavour jets with the CMS detector in pp collisions at $13 \mathrm{TeV}$, J. Instrum. 13, P05011 (2018).

[36] CMS Collaboration, Determination of jet energy calibration and transverse momentum resolution in CMS, J. Instrum. 6, P11002 (2011).

[37] CMS Collaboration, CMS luminosity measurements for the 2016 data taking period, CMS Physics Analysis Summary CMS-PAS-LUM-17-001, CERN, 2017, https://cds.cern.ch/ record/2257069.

[38] J. Butterworth et al., PDF4LHC recommendations for LHC Run II, J. Phys. G 43, 023001 (2016).

[39] M. Tanabashi et al. (Particle Data Group), Review of particle physics, Phys. Rev. D 98, 030001 (2018). 
A. M. Sirunyan, ${ }^{1, a}$ A. Tumasyan, ${ }^{1}$ W. Adam, ${ }^{2}$ F. Ambrogi, ${ }^{2}$ T. Bergauer, ${ }^{2}$ J. Brandstetter, ${ }^{2}$ M. Dragicevic, ${ }^{2}$ J. Erö, ${ }^{2}$ A. Escalante Del Valle, ${ }^{2}$ M. Flechl, ${ }^{2}$ R. Frühwirth, ${ }^{2, b}$ M. Jeitler, ${ }^{2, b}$ N. Krammer, ${ }^{2}$ I. Krätschmer, ${ }^{2}$ D. Liko, ${ }^{2}$ T. Madlener, ${ }^{2}$ I. Mikulec, ${ }^{2}$ N. Rad, ${ }^{2}$ J. Schieck, ${ }^{2, b}$ R. Schöfbeck, ${ }^{2}$ M. Spanring, ${ }^{2}$ D. Spitzbart, ${ }^{2}$ W. Waltenberger, ${ }^{2}$ C.-E. Wulz, ${ }^{2, b}$ M. Zarucki, ${ }^{2}$ V. Drugakov, ${ }^{3}$ V. Mossolov, ${ }^{3}$ J. Suarez Gonzalez, ${ }^{3}$ M. R. Darwish, ${ }^{4}$ E. A. De Wolf, ${ }^{4}$ D. Di Croce, ${ }^{4}$ X. Janssen, ${ }^{4}$ J. Lauwers, ${ }^{4}$ A. Lelek, ${ }^{4}$ M. Pieters, ${ }^{4}$ H. Rejeb Sfar, ${ }^{4}$ H. Van Haevermaet, ${ }^{4}$ P. Van Mechelen, ${ }^{4}$ S. Van Putte, ${ }^{4}$ N. Van Remortel, ${ }^{4}$ F. Blekman, ${ }^{5}$ E. S. Bols, ${ }^{5}$ S. S. Chhibra, ${ }^{5}$ J. D’Hondt, ${ }^{5}$ J. De Clercq, ${ }^{5}$ D. Lontkovskyi, ${ }^{5}$ S. Lowette, ${ }^{5}$ I. Marchesini ${ }^{5}$ S. Moortgat, ${ }^{5}$ L. Moreels, ${ }^{5}$ Q. Python,${ }^{5}$ K. Skovpen,${ }^{5}$ S. Tavernier, ${ }^{5}$ W. Van Doninck, ${ }^{5}$ P. Van Mulders, ${ }^{5}$ I. Van Parijs, ${ }^{5}$ D. Beghin, ${ }^{6}$ B. Bilin, ${ }^{6}$ H. Brun, ${ }^{6}$ B. Clerbaux, ${ }^{6}$ G. De Lentdecker, ${ }^{6}$ H. Delannoy, ${ }^{6}$ B. Dorney,${ }^{6}$ L. Favart, ${ }^{6}$ A. Grebenyuk, ${ }^{6}$ A. K. Kalsi, ${ }^{6}$ J. Luetic, ${ }^{6}$ A. Popov,${ }^{6}$ N. Postiau, ${ }^{6}$ E. Starling, ${ }^{6}$ L. Thomas, ${ }^{6}$ C. Vander Velde, ${ }^{6}$ P. Vanlaer, ${ }^{6}$ D. Vannerom, ${ }^{6}$ Q. Wang, ${ }^{6}$ T. Cornelis, ${ }^{7}$ D. Dobur, ${ }^{7}$ I. Khvastunov, ${ }^{7, c}$ C. Roskas, ${ }^{7}$ D. Trocino, ${ }^{7}$ M. Tytgat, ${ }^{7}$ W. Verbeke, ${ }^{7}$ B. Vermassen, ${ }^{7}$ M. Vit, ${ }^{7}$ N. Zaganidis, ${ }^{7}$ O. Bondu,${ }^{8}$ G. Bruno, ${ }^{8}$ C. Caputo, ${ }^{8}$ P. David,${ }^{8}$ C. Delaere,${ }^{8}$ M. Delcourt, ${ }^{8}$ A. Giammanco, ${ }^{8}$ V. Lemaitre, ${ }^{8}$ A. Magitteri, ${ }^{8}$ J. Prisciandaro, ${ }^{8}$ A. Saggio, ${ }^{8}$ M. Vidal Marono,${ }^{8}$ P. Vischia, ${ }^{8}$ J. Zobec ${ }^{8}$ F. L. Alves, ${ }^{9}$ G. A. Alves, ${ }^{9}$ G. Correia Silva, ${ }^{9}$ C. Hensel,,${ }^{9}$ A. Moraes, ${ }^{9}$ P. Rebello Teles, ${ }^{9}$ E. Belchior Batista Das Chagas, ${ }^{10}$ W. Carvalho, ${ }^{10}$ J. Chinellato, ${ }^{10, \mathrm{~d}}$ E. Coelho, ${ }^{10}$ E. M. Da Costa, ${ }^{10}$ G. G. Da Silveira, ${ }^{10, \mathrm{e}}$ D. De Jesus Damiao, ${ }^{10}$ C. De Oliveira Martins, ${ }^{10}$ S. Fonseca De Souza, ${ }^{10}$ L. M. Huertas Guativa, ${ }^{10}$ H. Malbouisson, ${ }^{10}$ J. Martins,,${ }^{10, f}$ D. Matos Figueiredo, ${ }^{10}$ M. Medina Jaime, ${ }^{10, \mathrm{~g}}$ M. Melo De Almeida, ${ }^{10}$ C. Mora Herrera,${ }^{10}$ L. Mundim,${ }^{10}$ H. Nogima, ${ }^{10}$ W. L. Prado Da Silva, ${ }^{10}$ L. J. Sanchez Rosas, ${ }^{10}$ A. Santoro, ${ }^{10}$ A. Sznajder ${ }^{10}$ M. Thiel,${ }^{10}$ E. J. Tonelli Manganote, ${ }^{10, d}$ F. Torres Da Silva De Araujo, ${ }^{10}$ A. Vilela Pereira, ${ }^{10}$ S. Ahuja, ${ }^{11 a}$ C. A. Bernardes, ${ }^{11 a}$ L. Calligaris, ${ }^{11 a}$

T. R. Fernandez Perez Tomei, ${ }^{11 \mathrm{a}}$ E. M. Gregores, ${ }^{11 \mathrm{a}, 1 \mathrm{~b}}$ D. S. Lemos, ${ }^{11 \mathrm{a}}$ P. G. Mercadante, ${ }^{11 \mathrm{a}, 11 \mathrm{~b}}$ S. F. Novaes, ${ }^{11 \mathrm{a}}$ Sandra S. Padula, ${ }^{11 a}$ A. Aleksandrov, ${ }^{12}$ G. Antchev, ${ }^{12}$ R. Hadjiiska, ${ }^{12}$ P. Iaydjiev, ${ }^{12}$ A. Marinov, ${ }^{12}$ M. Misheva, ${ }^{12}$ M. Rodozov, ${ }^{12}$ M. Shopova, ${ }^{12}$ G. Sultanov, ${ }^{12}$ M. Bonchev, ${ }^{13}$ A. Dimitrov, ${ }^{13}$ T. Ivanov, ${ }^{13}$ L. Litov, ${ }^{13}$ B. Pavlov, ${ }^{13}$ P. Petkov, ${ }^{13}$ W. Fang, ${ }^{14, h}$ X. Gao, ${ }^{14, h}$ L. Yuan, ${ }^{14}$ M. Ahmad, ${ }^{15}$ G. M. Chen, ${ }^{15}$ H. S. Chen, ${ }^{15}$ M. Chen, ${ }^{15}$ C. H. Jiang, ${ }^{15}$ D. Leggat,${ }^{15}$ H. Liao, ${ }^{15}$ Z. Liu, ${ }^{15}$ S. M. Shaheen, ${ }^{15, i}$ A. Spiezia, ${ }^{15}$ J. Tao, ${ }^{15}$ E. Yazgan, ${ }^{15}$ H. Zhang, ${ }^{15}$ S. Zhang, ${ }^{15, i}$ J. Zhao, ${ }^{15}$ A. Agapitos, ${ }^{16}$ Y. Ban, ${ }^{16}$ G. Chen, ${ }^{16}$ A. Levin, ${ }^{16}$ J. Li ${ }^{16}$ L. Li,${ }^{16}$ Q. Li,${ }^{16}$ Y. Mao, ${ }^{16}$ S. J. Qian, ${ }^{16}$ D. Wang, ${ }^{16}$ Z. Hu, ${ }^{17}$ Y. Wang, ${ }^{17}$ C. Avila, ${ }^{18}$ A. Cabrera, ${ }^{18}$ L. F. Chaparro Sierra, ${ }^{18}$ C. Florez, ${ }^{18}$ C. F. González Hernández,${ }^{18}$ M. A. Segura Delgado, ${ }^{18}$ J. Mejia Guisao, ${ }^{19}$ J. D. Ruiz Alvarez, ${ }^{19}$ C. A. Salazar González, ${ }^{19}$ N. Vanegas Arbelaez, ${ }^{19}$ D. Giljanović, ${ }^{20}$ N. Godinovic,${ }^{20}$ D. Lelas, ${ }^{20}$ I. Puljak ${ }^{20}$ T. Sculac, ${ }^{20}$ Z. Antunovic, ${ }^{21}$ M. Kovac,${ }^{21}$ V. Brigljevic, ${ }^{22}$ S. Ceci, ${ }^{22}$ D. Ferencek, ${ }^{22}$ K. Kadija, ${ }^{22}$ B. Mesic,${ }^{22}$ M. Roguljic, ${ }^{22}$ A. Starodumov, ${ }^{22, j}$ T. Susa, ${ }^{22}$ M. W. Ather, ${ }^{23}$ A. Attikis, ${ }^{23}$ E. Erodotou, ${ }^{23}$ A. Ioannou, ${ }^{23}$ M. Kolosova, ${ }^{23}$ S. Konstantinou, ${ }^{23}$ G. Mavromanolakis, ${ }^{23}$ J. Mousa, ${ }^{23}$ C. Nicolaou, ${ }^{23}$ F. Ptochos, ${ }^{23}$ P. A. Razis, ${ }^{23}$ H. Rykaczewski, ${ }^{23}$ D. Tsiakkouri, ${ }^{23}$ M. Finger, ${ }^{24, k}$ M. Finger Jr., ${ }^{24, k}$ A. Kveton, ${ }^{24}$ J. Tomsa, ${ }^{24}$ E. Ayala, ${ }^{25}$ E. Carrera Jarrin, ${ }^{26}$ S. Abu Zeid,,${ }^{27,1}$ S. Khalil, ${ }^{27, \mathrm{~m}}$ S. Bhowmik, ${ }^{28}$ A. Carvalho Antunes De Oliveira, ${ }^{28}$ R. K. Dewanjee, ${ }^{28} \mathrm{~K}$. Ehataht, ${ }^{28} \mathrm{M}$. Kadastik, ${ }^{28}$ M. Raidal ${ }^{28}$ C. Veelken, ${ }^{28}$ P. Eerola, ${ }^{29}$ L. Forthomme, ${ }^{29}$ H. Kirschenmann, ${ }^{29}$ K. Osterberg, ${ }^{29}$ M. Voutilainen, ${ }^{29}$ F. Garcia, ${ }^{30}$

J. Havukainen, ${ }^{30}$ J. K. Heikkilä, ${ }^{30}$ T. Järvinen, ${ }^{30}$ V. Karimäki, ${ }^{30}$ R. Kinnunen ${ }^{30}$ T. Lampén, ${ }^{30}$ K. Lassila-Perini, ${ }^{30}$ S. Laurila, ${ }^{30}$ S. Lehti, ${ }^{30}$ T. Lindén, ${ }^{30}$ P. Luukka,${ }^{30}$ T. Mäenpää, ${ }^{30}$ H. Siikonen,,${ }^{30}$ E. Tuominen,${ }^{30}$ J. Tuominiemi,${ }^{30}$ T. Tuuva,${ }^{31}$ M. Besancon, ${ }^{32}$ F. Couderc, ${ }^{32}$ M. Dejardin, ${ }^{32}$ D. Denegri, ${ }^{32}$ B. Fabbro, ${ }^{32}$ J. L. Faure, ${ }^{32}$ F. Ferri, ${ }^{32}$ S. Ganjour, ${ }^{32}$ A. Givernaud ${ }^{32}$ P. Gras, ${ }^{32}$ G. Hamel de Monchenault, ${ }^{32}$ P. Jarry, ${ }^{32}$ C. Leloup, ${ }^{32}$ E. Locci, ${ }^{32}$ J. Malcles, ${ }^{32}$ J. Rander, ${ }^{32}$ A. Rosowsky, ${ }^{32}$ M. Ö. Sahin, ${ }^{32}$ A. Savoy-Navarro, ${ }^{32, n}$ M. Titov, ${ }^{32}$ C. Amendola,${ }^{33}$ F. Beaudette, ${ }^{33}$ P. Busson, ${ }^{33}$ C. Charlot,${ }^{33}$ B. Diab ${ }^{33}$ G. Falmagne, ${ }^{33}$ R. Granier de Cassagnac, ${ }^{33}$ I. Kucher ${ }^{33}$ A. Lobanov, ${ }^{33}$ C. Martin Perez, ${ }^{33}$ M. Nguyen, ${ }^{33}$ C. Ochando, ${ }^{33}$ P. Paganini, ${ }^{33}$ J. Rembser,${ }^{33}$ R. Salerno, ${ }^{33}$ J. B. Sauvan, ${ }^{33}$ Y. Sirois, ${ }^{33}$ A. Zabi, ${ }^{33}$ A. Zghiche, ${ }^{33}$ J.-L. Agram, ${ }^{34,0}$ J. Andrea, ${ }^{34}$ D. Bloch,${ }^{34}$ G. Bourgatte, ${ }^{34}$ J.-M. Brom, ${ }^{34}$ E. C. Chabert, ${ }^{34}$ C. Collard, ${ }^{34}$ E. Conte, ${ }^{34,0}$ J.-C. Fontaine, ${ }^{34,0}$ D. Gelé, ${ }^{34}$ U. Goerlach, ${ }^{34}$ M. Jansová, ${ }^{34}$ A.-C. Le Bihan, ${ }^{34}$ N. Tonon, ${ }^{34}$ P. Van Hove, ${ }^{34}$ S. Gadrat,${ }^{35}$ S. Beauceron, ${ }^{36}$ C. Bernet, ${ }^{36}$ G. Boudoul,${ }^{36}$ C. Camen, ${ }^{36}$ N. Chanon,${ }^{36}$ R. Chierici, ${ }^{36}$ D. Contardo,${ }^{36}$ P. Depasse, ${ }^{36}$ H. El Mamouni, ${ }^{36}$ J. Fay,${ }^{36}$ S. Gascon, ${ }^{36}$ M. Gouzevitch, ${ }^{36}$ B. Ille, ${ }^{36}$ Sa. Jain, ${ }^{36}$ F. Lagarde, ${ }^{36}$ I. B. Laktineh, ${ }^{36}$ H. Lattaud,${ }^{36}$ M. Lethuillier, ${ }^{36}$

L. Mirabito, ${ }^{36}$ S. Perries, ${ }^{36}$ V. Sordini, ${ }^{36}$ G. Touquet,${ }^{36}$ M. Vander Donckt, ${ }^{36}$ S. Viret, ${ }^{36}$ A. Khvedelidze,${ }^{37, k}$ Z. Tsamalaidze, ${ }^{38, k}$ C. Autermann, ${ }^{39}$ L. Feld, ${ }^{39}$ M. K. Kiesel, ${ }^{39}$ K. Klein, ${ }^{39}$ M. Lipinski, ${ }^{39}$ D. Meuser, ${ }^{39}$ A. Pauls, ${ }^{39}$ M. Preuten, ${ }^{39}$ M. P. Rauch, ${ }^{39}$ C. Schomakers, ${ }^{39}$ J. Schulz, ${ }^{39}$ M. Teroerde, ${ }^{39}$ B. Wittmer, ${ }^{39}$ A. Albert,${ }^{40}$ M. Erdmann, ${ }^{40}$ S. Erdweg, ${ }^{40}$ T. Esch, ${ }^{40}$ B. Fischer, ${ }^{40}$ R. Fischer, ${ }^{40}$ S. Ghosh, ${ }^{40}$ T. Hebbeker, ${ }^{40}$ K. Hoepfner, ${ }^{40}$ H. Keller,${ }^{40}$ L. Mastrolorenzo, ${ }^{40}$ M. Merschmeyer, ${ }^{40}$ A. Meyer, ${ }^{40}$ P. Millet,${ }^{40}$ G. Mocellin,${ }^{40}$ S. Mondal, ${ }^{40}$ S. Mukherjee ${ }^{40}$ D. Noll, ${ }^{40}$ A. Novak, ${ }^{40}$ T. Pook, ${ }^{40}$ A. Pozdnyakov, ${ }^{40}$ T. Quast, ${ }^{40}$ M. Radziej, ${ }^{40}$ Y. Rath, ${ }^{40}$ H. Reithler, ${ }^{40}$ M. Rieger, ${ }^{40}$ J. Roemer, ${ }^{40}$ 
A. Schmidt ${ }^{40}$ S. C. Schuler, ${ }^{40}$ A. Sharma, ${ }^{40}$ S. Thüer, ${ }^{40}$ S. Wiedenbeck,${ }^{40}$ G. Flügge,${ }^{41}$ W. Haj Ahmad, ${ }^{41, p}$ O. Hlushchenko, ${ }^{41}$ T. Kress, ${ }^{41}$ T. Müller, ${ }^{41}$ A. Nehrkorn, ${ }^{41}$ A. Nowack, ${ }^{41}$ C. Pistone, ${ }^{41}$ O. Pooth, ${ }^{41}$ D. Roy, ${ }^{41}$ H. Sert, ${ }^{41}$ A. Stahl,${ }^{41, q}$ M. Aldaya Martin, ${ }^{42}$ P. Asmuss, ${ }^{42}$ I. Babounikau, ${ }^{42}$ H. Bakhshiansohi, ${ }^{42}$ K. Beernaert ${ }^{42}$ O. Behnke, ${ }^{42}$ U. Behrens, ${ }^{42}$ A. Bermúdez Martínez, ${ }^{42}$ D. Bertsche, ${ }^{42}$ A. A. Bin Anuar, ${ }^{42}$ K. Borras, ${ }^{42, \mathrm{r}}$ V. Botta ${ }^{42}$ A. Campbell, ${ }^{42}$ A. Cardini, ${ }^{42}$ P. Connor, ${ }^{42}$ S. Consuegra Rodríguez, ${ }^{42}$ C. Contreras-Campana, ${ }^{42}$ V. Danilov, ${ }^{42}$ A. De Wit, ${ }^{42}$ M. M. Defranchis, ${ }^{42}$ C. Diez Pardos, ${ }^{42}$ D. Domínguez Damiani ${ }^{42}$ G. Eckerlin,${ }^{42}$ D. Eckstein, ${ }^{42}$ T. Eichhorn, ${ }^{42}$ A. Elwood, ${ }^{42}$ E. Eren, ${ }^{42}$ E. Gallo, ${ }^{42, s}$ A. Geiser, ${ }^{42}$ J. M. Grados Luyando, ${ }^{42}$ A. Grohsjean, ${ }^{42}$ M. Guthoff, ${ }^{42}$ M. Haranko, ${ }^{42}$ A. Harb, ${ }^{42}$ A. Jafari, ${ }^{42}$ N. Z. Jomhari, ${ }^{42}$ H. Jung, ${ }^{42}$ A. Kasem, ${ }^{42, \mathrm{r}}$ M. Kasemann, ${ }^{42}$ H. Kaveh, ${ }^{42}$ J. Keaveney, ${ }^{42}$ C. Kleinwort, ${ }^{42}$ J. Knolle, ${ }^{42}$ D. Krücker, ${ }^{42}$ W. Lange, ${ }^{42}$ T. Lenz, ${ }^{42}$ J. Leonard, ${ }^{42}$ J. Lidrych, ${ }^{42}$ K. Lipka, ${ }^{42}$ W. Lohmann, ${ }^{42, t}$ R. Mankel, ${ }^{42}$ I.-A. Melzer-Pellmann, ${ }^{42}$ A. B. Meyer ${ }^{42}$ M. Meyer, ${ }^{42}$ M. Missiroli, ${ }^{42}$ G. Mittag, ${ }^{42}$ J. Mnich, ${ }^{42}$ A. Mussgiller ${ }^{42}$ V. Myronenko, ${ }^{42}$ D. Pérez Adán, ${ }^{42}$ S. K. Pflitsch, ${ }^{42}$ D. Pitzl ${ }^{42}$ A. Raspereza, ${ }^{42}$ A. Saibel, ${ }^{42}$ M. Savitskyi, ${ }^{42}$ V. Scheurer ${ }^{42}$

P. Schütze, ${ }^{42}$ C. Schwanenberger, ${ }^{42}$ R. Shevchenko, ${ }^{42}$ A. Singh ${ }^{42}$ H. Tholen,${ }^{42}$ O. Turkot,${ }^{42}$ A. Vagnerini, ${ }^{42}$ M. Van De Klundert, ${ }^{42}$ G. P. Van Onsem, ${ }^{42}$ R. Walsh,${ }^{42}$ Y. Wen, ${ }^{42}$ K. Wichmann, ${ }^{42}$ C. Wissing, ${ }^{42}$ O. Zenaiev, ${ }^{42}$ R. Zlebcik, ${ }^{42}$ R. Aggleton, ${ }^{43}$ S. Bein, ${ }^{43}$ L. Benato, ${ }^{43}$ A. Benecke, ${ }^{43}$ V. Blobel, ${ }^{43}$ T. Dreyer,${ }^{43}$ A. Ebrahimi, ${ }^{43}$ A. Fröhlich,${ }^{43}$ C. Garbers,${ }^{43}$ E. Garutti, ${ }^{43}$ D. Gonzalez, ${ }^{43}$ P. Gunnellini, ${ }^{43}$ J. Haller, ${ }^{43}$ A. Hinzmann, ${ }^{43}$ A. Karavdina, ${ }^{43}$ G. Kasieczka, ${ }^{43}$ R. Klanner, ${ }^{43}$ R. Kogler, ${ }^{43}$ N. Kovalchuk, ${ }^{43}$ S. Kurz, ${ }^{43}$ V. Kutzner, ${ }^{43}$ J. Lange, ${ }^{43}$ T. Lange, ${ }^{43}$ A. Malara, ${ }^{43}$ D. Marconi, ${ }^{43}$ J. Multhaup ${ }^{43}$ M. Niedziela, ${ }^{43}$ C. E. N. Niemeyer, ${ }^{43}$ D. Nowatschin, ${ }^{43}$ A. Perieanu, ${ }^{43}$ A. Reimers, ${ }^{43}$ O. Rieger,${ }^{43}$ C. Scharf,${ }^{43}$ P. Schleper, ${ }^{43}$ S. Schumann, ${ }^{43}$ J. Schwandt, ${ }^{43}$ J. Sonneveld, ${ }^{43}$ H. Stadie, ${ }^{43}$ G. Steinbrück, ${ }^{43}$ F. M. Stober ${ }^{43}$ M. Stöver ${ }^{43}$ B. Vormwald, ${ }^{43}$ I. Zoi, ${ }^{43}$ M. Akbiyik, ${ }^{44}$ C. Barth, ${ }^{44}$ M. Baselga, ${ }^{44}$ S. Baur, ${ }^{44}$ T. Berger, ${ }^{44}$ E. Butz,${ }^{44}$ R. Caspart, ${ }^{44}$ T. Chwalek, ${ }^{44}$ W. De Boer,${ }^{44}$ A. Dierlamm, ${ }^{44}$ K. El Morabit, ${ }^{44}$ N. Faltermann, ${ }^{44}$ M. Giffels, ${ }^{44}$ P. Goldenzweig, ${ }^{44}$ A. Gottmann, ${ }^{44}$ M. A. Harrendorf, ${ }^{44}$ F. Hartmann, ${ }^{44, q}$ U. Husemann, ${ }^{44}$ S. Kudella, ${ }^{44}$ S. Mitra, ${ }^{44}$ M. U. Mozer, ${ }^{44}$ Th. Müller, ${ }^{44}$ M. Musich, ${ }^{44}$ A. Nürnberg, ${ }^{44}$ G. Quast, ${ }^{44}$ K. Rabbertz, ${ }^{44}$ M. Schröder, ${ }^{44}$ I. Shvetsov, ${ }^{44}$ H. J. Simonis, ${ }^{44}$ R. Ulrich,${ }^{44}$ M. Weber ${ }^{44}$ C. Wöhrmann, ${ }^{44}$ R. Wolf ${ }^{44}$ G. Anagnostou, ${ }^{45}$ P. Asenov, ${ }^{45}$ G. Daskalakis, ${ }^{45}$ T. Geralis, ${ }^{45}$ A. Kyriakis, ${ }^{45}$ D. Loukas, ${ }^{45}$ G. Paspalaki, ${ }^{45}$ M. Diamantopoulou ${ }^{46}$ G. Karathanasis, ${ }^{46}$ P. Kontaxakis, ${ }^{46}$ A. Panagiotou, ${ }^{46}$ I. Papavergou, ${ }^{46}$ N. Saoulidou, ${ }^{46}$ A. Stakia, ${ }^{46}$

K. Theofilatos, ${ }^{46}$ K. Vellidis, ${ }^{46}$ G. Bakas, ${ }^{47}$ K. Kousouris, ${ }^{47}$ I. Papakrivopoulos,${ }^{47}$ G. Tsipolitis, ${ }^{47}$ I. Evangelou, ${ }^{48}$

C. Foudas, ${ }^{48}$ P. Gianneios, ${ }^{48}$ P. Katsoulis, ${ }^{48}$ P. Kokkas, ${ }^{48}$ S. Mallios, ${ }^{48}$ K. Manitara, ${ }^{48}$ N. Manthos, ${ }^{48}$ I. Papadopoulos, ${ }^{48}$

J. Strologas, ${ }^{48}$ F. A. Triantis, ${ }^{48}$ D. Tsitsonis, ${ }^{48}$ M. Bartók, ${ }^{49, u}$ M. Csanad, ${ }^{49}$ P. Major, ${ }^{49}$ K. Mandal, ${ }^{49}$ A. Mehta, ${ }^{49}$ M. I. Nagy, ${ }^{49}$ G. Pasztor, ${ }^{49}$ O. Surányi,${ }^{49}$ G. I. Veres, ${ }^{49}$ G. Bencze, ${ }^{50}$ C. Hajdu,${ }^{50}$ D. Horvath,${ }^{50, v}$ F. Sikler, ${ }^{50}$ T. Á. Vámi, ${ }^{50}$ V. Veszpremi, ${ }^{50}$ G. Vesztergombi,${ }^{50, a, w}$ N. Beni ${ }^{51}$ S. Czellar, ${ }^{51}$ J. Karancsi,${ }^{51, u}$ A. Makovec, ${ }^{51}$ J. Molnar, ${ }^{51}$ Z. Szillasi, ${ }^{51}$ P. Raics, ${ }^{52}$ D. Teyssier, ${ }^{52}$ Z. L. Trocsanyi ${ }^{52}$ B. Ujvari, ${ }^{52}$ T. Csorgo, ${ }^{53}$ W. J. Metzger, ${ }^{53}$ F. Nemes ${ }^{53}$ T. Novak, ${ }^{53}$ S. Choudhury, ${ }^{54}$ J. R. Komaragiri, ${ }^{54}$ P. C. Tiwari, ${ }^{54}$ S. Bahinipati, ${ }^{55, x}$ C. Kar, ${ }^{55}$ G. Kole, ${ }^{55}$ P. Mal, ${ }^{55}$

V. K. Muraleedharan Nair Bindhu, ${ }^{55}$ A. Nayak, ${ }^{55, y}$ D. K. Sahoo,${ }^{55, x}$ S. K. Swain, ${ }^{55}$ S. Bansal,${ }^{56}$ S. B. Beri, ${ }^{56}$ V. Bhatnagar, ${ }^{56}$ S. Chauhan, ${ }^{56}$ R. Chawla, ${ }^{56}$ N. Dhingra, ${ }^{56}$ R. Gupta, ${ }^{56}$ A. Kaur, ${ }^{56}$ M. Kaur, ${ }^{56}$ S. Kaur, ${ }^{56}$ P. Kumari, ${ }^{56}$ M. Lohan, ${ }^{56}$ M. Meena, ${ }^{56}$ K. Sandeep, ${ }^{56}$ S. Sharma, ${ }^{56}$ J. B. Singh, ${ }^{56}$ A. K. Virdi, ${ }^{56}$ G. Walia, ${ }^{56}$ A. Bhardwaj, ${ }^{57}$ B. C. Choudhary, ${ }^{57}$ R. B. Garg, ${ }^{57}$ M. Gola ${ }^{57}$ S. Keshri, ${ }^{57}$ Ashok Kumar, ${ }^{57}$ S. Malhotra, ${ }^{57}$ M. Naimuddin, ${ }^{57}$ P. Priyanka, ${ }^{57}$ K. Ranjan, ${ }^{57}$ Aashaq Shah, ${ }^{57}$ R. Sharma, ${ }^{57}$ R. Bhardwaj, ${ }^{58, z}$ M. Bharti, ${ }^{58, z}$ R. Bhattacharya, ${ }^{58}$ S. Bhattacharya, ${ }^{58}$ U. Bhawandeep, ${ }^{58, z}$ D. Bhowmik, ${ }^{58}$ S. Dey, ${ }^{58}$ S. Dutta, ${ }^{58}$ S. Ghosh, ${ }^{58}$ M. Maity, ${ }^{58, \text { aa }}$ K. Mondal, ${ }^{58}$ S. Nandan, ${ }^{58}$ A. Purohit, ${ }^{58}$ P. K. Rout,${ }^{58}$ G. Saha, ${ }^{58}$ S. Sarkar, ${ }^{58}$ T. Sarkar, ${ }^{58, a a}$ M. Sharan, ${ }^{58}$ B. Singh, ${ }^{58, z}$ S. Thakur, ${ }^{58, z}$ P. K. Behera, ${ }^{59}$ P. Kalbhor, ${ }^{59}$ A. Muhammad,${ }^{59}$

P. R. Pujahari, ${ }^{59}$ A. Sharma, ${ }^{59}$ A. K. Sikdar, ${ }^{59}$ R. Chudasama, ${ }^{60}$ D. Dutta, ${ }^{60}$ V. Jha, ${ }^{60}$ V. Kumar, ${ }^{60}$ D. K. Mishra, ${ }^{60}$ P. K. Netrakanti, ${ }^{60}$ L. M. Pant, ${ }^{60}$ P. Shukla, ${ }^{60}$ T. Aziz, ${ }^{61}$ M. A. Bhat, ${ }^{61}$ S. Dugad,${ }^{61}$ G. B. Mohanty, ${ }^{61}$ N. Sur, ${ }^{61}$ Ravindra Kumar Verma, ${ }^{61}$ S. Banerjee, ${ }^{62}$ S. Bhattacharya, ${ }^{62}$ S. Chatterjee, ${ }^{62}$ P. Das, ${ }^{62}$ M. Guchait, ${ }^{62}$ S. Karmakar, ${ }^{62}$ S. Kumar, ${ }^{62}$ G. Majumder, ${ }^{62}$ K. Mazumdar, ${ }^{62}$ N. Sahoo, ${ }^{62}$ S. Sawant,${ }^{62}$ S. Chauhan, ${ }^{63}$ S. Dube, ${ }^{63}$ V. Hegde, ${ }^{63}$ A. Kapoor,${ }^{63}$

K. Kothekar, ${ }^{63}$ S. Pandey, ${ }^{63}$ A. Rane,${ }^{63}$ A. Rastogi, ${ }^{63}$ S. Sharma, ${ }^{63}$ S. Chenarani, ${ }^{64, b b}$ E. Eskandari Tadavani, ${ }^{64}$

S. M. Etesami, ${ }^{64, b b}$ M. Khakzad, ${ }^{64}$ M. Mohammadi Najafabadi, ${ }^{64}$ M. Naseri, ${ }^{64}$ F. Rezaei Hosseinabadi, ${ }^{64}$ M. Felcini, ${ }^{65}$ M. Grunewald ${ }^{65}$ M. Abbrescia, ${ }^{66 a, 66 b}$ C. Calabria ${ }^{66 a, 66 b}$ A. Colaleo, ${ }^{66 a}$ D. Creanza, ${ }^{66 a, 66 c}$ L. Cristella, ${ }^{66 a, 66 b}$

N. De Filippis, ${ }^{66,66 \mathrm{c}}$ M. De Palma, ${ }^{66 a, 66 \mathrm{~b}}$ A. Di Florio, ${ }^{66 a, 66 \mathrm{~b}}$ L. Fiore, ${ }^{66 \mathrm{a}}$ A. Gelmi, ${ }^{66 a, 66 \mathrm{~b}}$ G. Iaselli, ${ }^{66 a, 66 c}$ M. Ince,${ }^{66 a, 66 \mathrm{~b}}$ S. Lezki, ${ }^{66 a, 66 b}$ G. Maggi, ${ }^{66 a, 66 c}$ M. Maggi, ${ }^{66 a}$ G. Miniello, ${ }^{66 a, 66 b}$ S. My, ${ }^{66 a, 66 b}$ S. Nuzzo, ${ }^{66 a, 66 b}$ A. Pompili, ${ }^{66 a, 66 b}$ G. Pugliese, ${ }^{66 a, 66 \mathrm{c}}$ R. Radogna, ${ }^{66 \mathrm{a}}$ A. Ranieri, ${ }^{66 \mathrm{a}}$ G. Selvaggi, ${ }^{66 a, 66 \mathrm{~b}}$ L. Silvestris, ${ }^{66 \mathrm{a}}$ R. Venditti, ${ }^{66 \mathrm{a}}$ P. Verwilligen, ${ }^{66 \mathrm{a}}$ G. Abbiendi, ${ }^{67 \mathrm{a}}$ C. Battilana, ${ }^{67,67 \mathrm{~b}}$ D. Bonacorsi, ${ }^{67,67 \mathrm{~b}}$ L. Borgonovi, ${ }^{67 a, 67 b}$ S. Braibant-Giacomelli, ${ }^{67,67 \mathrm{~b}}$ 
R. Campanini, ${ }^{67,67 b}$ P. Capiluppi,${ }^{67 a, 67 b}$ A. Castro, ${ }^{67 a, 67 b}$ F. R. Cavallo, ${ }^{67 a}$ C. Ciocca,${ }^{67 a}$ G. Codispoti, ${ }^{67 a, 67 b}$ M. Cuffiani,${ }^{67 a, 67 b}$ G. M. Dallavalle, ${ }^{67 a}$ F. Fabbri, ${ }^{67 a}$ A. Fanfani, ${ }^{67 a, 67 b}$ E. Fontanesi,${ }^{67 a}$ P. Giacomelli, ${ }^{67 a}$ C. Grandi, ${ }^{67 a}$ L. Guiducci, ${ }^{67 a, 67 b}$ F. Iemmi, ${ }^{67 a, 67 b}$ S. Lo Meo, ${ }^{67 a, c c}$ S. Marcellini, ${ }^{67 a}$ G. Masetti, ${ }^{67 a}$ F. L. Navarria, ${ }^{67 a, 67 b}$ A. Perrotta, ${ }^{67 a}$ F. Primavera, ${ }^{67,67 b}$ A. M. Rossi ${ }^{67 a, 67 b}$ T. Rovelli, ${ }^{67 a, 67 b}$ G. P. Siroli, ${ }^{67 a, 67 b}$ N. Tosi, ${ }^{67 a}$ S. Albergo, ${ }^{68 a, 68 b, d d}$ S. Costa, ${ }^{68 a, 68 b}$ A. Di Mattia, ${ }^{68 a}$ R. Potenza, ${ }^{68 a, 68 b}$ A. Tricomi, ${ }^{68 a, 68 b, d d}$ C. Tuve, ${ }^{68 a, 68 b}$ G. Barbagli, ${ }^{69 a}$ R. Ceccarelli, ${ }^{69 a}$ K. Chatterjee, ${ }^{69 a, 69 b}$ V. Ciulli, ${ }^{69 a, 69 b}$ C. Civinini, ${ }^{69 a}$ R. D’Alessandro, ${ }^{69 a, 69 b}$ E. Focardi, ${ }^{69 a, 69 b}$ G. Latino, ${ }^{69 a}$ P. Lenzi, ${ }^{69 a, 69 b}$ M. Meschini, ${ }^{69 a}$ S. Paoletti, ${ }^{69 a}$ G. Sguazzoni ${ }^{69 a}$ D. Strom ${ }^{69 a}$ L. Viliani,${ }^{69 a}$ L. Benussi, ${ }^{70}$ S. Bianco, ${ }^{70}$ D. Piccolo,${ }^{70}$ M. Bozzo $,{ }^{71 a}, 71 b$ F. Ferro, ${ }^{71 a}$ R. Mulargia ${ }^{71 a, 71 b}$ E. Robutti, ${ }^{71 a}$ S. Tosi, ${ }^{71 a, 71 b}$ A. Benaglia, ${ }^{72 a}$ A. Beschi, ${ }^{72 a, 72 b}$ F. Brivio, ${ }^{72 a, 72 b}$ V. Ciriolo, ${ }^{72 a, 72 b, q}$ S. Di Guida, ${ }^{72 a, 72 b, q}$ M. E. Dinardo, ${ }^{72 a, 72 b}$ P. Dini, ${ }^{72 a}$ S. Fiorendi, ${ }^{72 a, 72 b}$ S. Gennai, ${ }^{72 a}$ A. Ghezzi, ${ }^{72 a, 72 b}$ P. Govoni, ${ }^{72 a, 72 b}$

L. Guzzi, ${ }^{72 a, 72 b}$ M. Malberti, ${ }^{72 a}$ S. Malvezzi, ${ }^{72 a}$ D. Menasce, ${ }^{72 a}$ F. Monti, ${ }^{72 a, 72 b}$ L. Moroni, ${ }^{72 a}$ G. Ortona, ${ }^{72 a, 72 b}$

M. Paganoni, ${ }^{72 a, 72 b}$ D. Pedrini, ${ }^{72 a}$ S. Ragazzi, ${ }^{72 a, 72 b}$ T. Tabarelli de Fatis, ${ }^{72 a, 72 b}$ D. Zuolo, ${ }^{72 a, 72 b}$ S. Buontempo, ${ }^{73 a}$ N. Cavallo, ${ }^{73 a, 73 c}$ A. De Iorio, ${ }^{73 a, 73 b}$ A. Di Crescenzo, ${ }^{73 a, 73 b}$ F. Fabozzi, ${ }^{73 a, 73 c}$ F. Fienga ${ }^{73 a}$ G. Galati, ${ }^{73 a}$ A. O. M. Iorio, ${ }^{73 a, 73 b}$ L. Lista, ${ }^{73 a, 73 b}$ S. Meola, ${ }^{73 a, 73 d, q}$ P. Paolucci, ${ }^{73 a, q}$ B. Rossi ${ }^{73 a}$ C. Sciacca, ${ }^{73 a, 73 b}$ E. Voevodina, ${ }^{73 a, 73 b}$ P. Azzi, ${ }^{74 a}$ N. Bacchetta, ${ }^{74 a}$ A. Boletti, ${ }^{7 a, 74 b}$ A. Bragagnolo, ${ }^{74 a}$ R. Carlin, ${ }^{74 a}, 74 b$ P. Checchia, ${ }^{74 a}$ P. De Castro Manzano, ${ }^{74 a}$ T. Dorigo, ${ }^{74 a}$ U. Dosselli, ${ }^{74 a}$ F. Gasparini, ${ }^{74 a, 74 b}$ U. Gasparini ${ }^{74 a, 74 b}$ A. Gozzelino, ${ }^{74 a}$ S. Y. Hoh, ${ }^{74 a}$ P. Lujan, ${ }^{74 a}$ M. Margoni, ${ }^{74 a, 74 b}$ A. T. Meneguzzo, ${ }^{74 a, 74 b}$ J. Pazzini, ${ }^{74 a, 74 b}$ N. Pozzobon, ${ }^{74 a, 74 b}$ M. Presilla, ${ }^{74 a, 74 b}$ P. Ronchese,${ }^{74 a, 74 b}$ R. Rossin, ${ }^{74 a, 74 b}$ F. Simonetto, ${ }^{74 a, 74 b}$ A. Tiko, ${ }^{74 a}$ M. Tosi, ${ }^{74 a, 74 b}$ M. Zanetti, ${ }^{74 a, 74 b}$ P. Zotto, ${ }^{74 a, 74 b}$ G. Zumerle, ${ }^{74 a, 74 b}$ A. Braghieri, ${ }^{75 a}$ P. Montagna, ${ }^{75 a, 75 b}$ S. P. Ratti, ${ }^{75 a, 75 b}$ V. Re ${ }^{75 a}$ M. Ressegotti ${ }^{75 a, 75 b}$ C. Riccardi, ${ }^{75 a, 75 b}$ P. Salvini, ${ }^{75 a}$ I. Vai, ${ }^{75 a, 75 b}$ P. Vitulo, ${ }^{75 a, 75 b}$ M. Biasini, ${ }^{76 a, 76 b}$ G. M. Bilei, ${ }^{76 a}$ C. Cecchi, ${ }^{76 a, 76 b}$ D. Ciangottini, ${ }^{76 a, 76 b}$ L. Fanò,${ }^{76 a, 76 b}$ P. Lariccia, ${ }^{76 a, 76 b}$ R. Leonardi, ${ }^{76 a, 76 b}$ E. Manoni, ${ }^{76 a}$ G. Mantovani, ${ }^{76 a, 76 b}$ V. Mariani, ${ }^{76 a, 76 b}$ M. Menichelli, ${ }^{76 a}$ A. Rossi, ${ }^{76 a, 76 b}$ A. Santocchia, ${ }^{76 a, 76 b}$ D. Spiga, ${ }^{76 a}$ K. Androsov, ${ }^{77 a}$ P. Azzurri, ${ }^{77 a}$ G. Bagliesi, ${ }^{77 a}$ V. Bertacchi, ${ }^{77 a, 77 c}$ L. Bianchini, ${ }^{77 a}$ T. Boccali, ${ }^{77 a}$ R. Castaldi, ${ }^{77 a}$ M. A. Ciocci, ${ }^{77 a, 77 b}$ R. Dell'Orso, ${ }^{77 a}$ G. Fedi ${ }^{77 a}$ L. Giannini, ${ }^{77 a, 77 c}$ A. Giassi,${ }^{77 a}$ M. T. Grippo, ${ }^{77 a}$ F. Ligabue $,{ }^{77 a}, 77 \mathrm{c}$ E. Manca, ${ }^{77 a, 77 c}$ G. Mandorli, ${ }^{77 a, 77 c}$ A. Messineo, ${ }^{77,77 b}$ F. Palla, ${ }^{77 a}$ A. Rizzi, ${ }^{77 a, 77 b}$ G. Rolandi, ${ }^{77 a, e e}$ S. Roy Chowdhury, ${ }^{77 a}$ A. Scribano, ${ }^{77 a}$ P. Spagnolo, ${ }^{77 a}$ R. Tenchini, ${ }^{77 a}$ G. Tonelli, ${ }^{77,77 b}$ N. Turini, ${ }^{77 a}$ A. Venturi, ${ }^{77 a}$ P. G. Verdini, ${ }^{77 a}$ F. Cavallari, ${ }^{78 a}$ M. Cipriani, ${ }^{78 a, 78 b}$ D. Del Re ${ }^{78 a, 78 b}$ E. Di Marco, ${ }^{78 a}, 78 b$ M. Diemoz ${ }^{78 a}$ E. Longo, ${ }^{78 a, 78 b}$ B. Marzocchi, ${ }^{78 a, 78 b}$ P. Meridiani, ${ }^{78 a}$ G. Organtini, ${ }^{78 a, 78 b}$ F. Pandolfi, ${ }^{78 \mathrm{a}}$ R. Paramatti, ${ }^{78 a, 78 b}$ C. Quaranta, ${ }^{78 a}, 78 \mathrm{~b}$ S. Rahatlou, ${ }^{78 a, 78 b}$ C. Rovelli, ${ }^{78 a}$ F. Santanastasio, ${ }^{78 a, 78 b}$ L. Soffi, ${ }^{78 a, 78 b}$ N. Amapane, ${ }^{79 a, 79 b}$ R. Arcidiacono,${ }^{79 a, 79 c}$ S. Argiro, ${ }^{79 a, 79 b}$ M. Arneodo,${ }^{79 a, 79 c}$ N. Bartosik, ${ }^{79 a}$ R. Bellan, ${ }^{79 a, 79 b}$ C. Biino, ${ }^{79 a}$ A. Cappati, ${ }^{79 a, 79 b}$ N. Cartiglia, ${ }^{79 a}$ S. Cometti, ${ }^{79 a}$ M. Costa, ${ }^{79 a, 79 b}$ R. Covarelli, ${ }^{79 a, 79 b}$ N. Demaria, ${ }^{79 a}$ B. Kiani, ${ }^{79 a, 79 b}$ C. Mariotti ${ }^{79 a}$ S. Maselli, ${ }^{79 a}$ E. Migliore, ${ }^{79 a, 79 b}$ V. Monaco, ${ }^{79 a, 79 b}$ E. Monteil, ${ }^{79 a, 79 b}$ M. Monteno ${ }^{79 a}$ M. M. Obertino, ${ }^{79 a, 79 b}$ L. Pacher, ${ }^{79 a, 79 b}$ N. Pastrone, ${ }^{79 a}$ M. Pelliccioni ${ }^{79 a}$ G. L. Pinna Angioni, ${ }^{79 a, 79 b}$ A. Romero, ${ }^{79 a, 79 b}$ M. Ruspa, ${ }^{79 a, 79 c}$ R. Sacchi,${ }^{79 a, 79 b}$ R. Salvatico, ${ }^{79 a, 79 b}$ V. Sola ${ }^{79 a}$ A. Solano, ${ }^{79 a, 79 b}$ D. Soldi, ${ }^{79 a, 79 b}$ A. Staiano, ${ }^{79 a}$ S. Belforte, ${ }^{80 a}$ V. Candelise, ${ }^{80 a, 80 b}$ M. Casarsa, ${ }^{80 a}$ F. Cossutti, ${ }^{80 a}$ A. Da Rold, ${ }^{80 a, 80 b}$ G. Della Ricca, ${ }^{80 a, 80 b}$ F. Vazzoler, ${ }^{80 a, 80 b}$ A. Zanetti, ${ }^{80 a}$ B. Kim, ${ }^{81}$ D. H. Kim, ${ }^{81}$ G. N. Kim, ${ }^{81}$ M. S. Kim, ${ }^{81}$ J. Lee, ${ }^{81}$ S. W. Lee, ${ }^{81}$ C. S. Moon,${ }^{81}$ Y. D. Oh ${ }^{81}$ S. I. Pak, ${ }^{81}$ S. Sekmen, ${ }^{81}$ D. C. Son, ${ }^{81}$ Y. C. Yang, ${ }^{81}$ H. Kim, ${ }^{82}$ D. H. Moon,${ }^{82}$ G. Oh ${ }^{82}$ B. Francois, ${ }^{83}$ T. J. Kim, ${ }^{83}$ J. Park, ${ }^{83}$ S. Cho, ${ }^{84}$ S. Choi ${ }^{84}$ Y. Go, ${ }^{84}$ D. Gyun, ${ }^{84}$ S. Ha, ${ }^{84}$ B. Hong,${ }^{84}$ K. Lee, ${ }^{84}$ K. S. Lee, ${ }^{84}$ J. Lim, ${ }^{84}$ J. Park, ${ }^{84}$ S. K. Park, ${ }^{84}$ Y. Roh, ${ }^{84}$ J. Goh, ${ }^{85}$ H. S. Kim ${ }^{86}$ J. Almond, ${ }^{87}$ J. H. Bhyun, ${ }^{87}$ J. Choi ${ }^{87}$ S. Jeon, ${ }^{87}$ J. Kim, ${ }^{87}$ J. S. Kim, ${ }^{87}$ H. Lee, ${ }^{87}$ K. Lee, ${ }^{87}$ S. Lee, ${ }^{87}$ K. Nam, ${ }^{87}$ M. Oh, ${ }^{87}$ S. B. Oh, ${ }^{87}$ B. C. Radburn-Smith, ${ }^{87}$ U. K. Yang, ${ }^{87}$ H. D. Yoo, ${ }^{87}$ I. Yoon, ${ }^{87}$ G. B. Yu, ${ }^{87}$ D. Jeon, ${ }^{88}$ H. Kim, ${ }^{88}$ J. H. Kim, ${ }^{88}$ J. S. H. Lee, ${ }^{88}$ I. C. Park, ${ }^{88}$ I. Watson, ${ }^{88}$ Y. Choi,${ }^{89}$ C. Hwang, ${ }^{89}$ Y. Jeong, ${ }^{89}$ J. Lee, ${ }^{89}$ Y. Lee,${ }^{89}$ I. Yu ${ }^{89}$ V. Veckalns,${ }^{90, f f}$ V. Dudenas, ${ }^{91}$ A. Juodagalvis, ${ }^{91}$ J. Vaitkus, ${ }^{91}$ Z. A. Ibrahim, ${ }^{92}$ F. Mohamad Idris, ${ }^{92, g g}$

W. A. T. Wan Abdullah, ${ }^{92}$ M. N. Yusli, ${ }^{92}$ Z. Zolkapli, ${ }^{92}$ J. F. Benitez, ${ }^{93}$ A. Castaneda Hernandez, ${ }^{93}$ J. A. Murillo Quijada, ${ }^{93}$ L. Valencia Palomo, ${ }^{93}$ H. Castilla-Valdez, ${ }^{94}$ E. De La Cruz-Burelo, ${ }^{94}$ I. Heredia-De La Cruz,${ }^{94, \text { hh }}$ R. Lopez-Fernandez, ${ }^{94}$ A. Sanchez-Hernandez,${ }^{94}$ S. Carrillo Moreno, ${ }^{95}$ C. Oropeza Barrera,${ }^{95}$ M. Ramirez-Garcia, ${ }^{95}$ F. Vazquez Valencia, ${ }^{95}$ J. Eysermans, ${ }^{96}$ I. Pedraza ${ }^{96}$ H. A. Salazar Ibarguen,${ }^{96}$ C. Uribe Estrada, ${ }^{96}$ A. Morelos Pineda,${ }^{97}$ N. Raicevic, ${ }^{98}$ D. Krofcheck, ${ }^{99}$ S. Bheesette, ${ }^{100}$ P. H. Butler, ${ }^{100}$ A. Ahmad, ${ }^{101}$ M. Ahmad,${ }^{101}$ Q. Hassan, ${ }^{101}$ H. R. Hoorani,${ }^{101}$ W. A. Khan, ${ }^{101}$ M. A. Shah, ${ }^{101}$ M. Shoaib, ${ }^{101}$ M. Waqas, ${ }^{101}$ V. Avati, ${ }^{102}$ L. Grzanka, ${ }^{102}$ M. Malawski, ${ }^{102}$ H. Bialkowska, ${ }^{103}$ M. Bluj, ${ }^{103}$ B. Boimska, ${ }^{103}$ M. Górski, ${ }^{103}$ M. Kazana, ${ }^{103}$ M. Szleper, ${ }^{103}$ P. Zalewski, ${ }^{103}$ K. Bunkowski, ${ }^{104}$ A. Byszuk,,${ }^{104, i i}$ K. Doroba, ${ }^{104}$ A. Kalinowski, ${ }^{104}$ M. Konecki, ${ }^{104}$ J. Krolikowski, ${ }^{104}$ M. Misiura, ${ }^{104}$ M. Olszewski, ${ }^{104}$ A. Pyskir, ${ }^{104}$ M. Walczak, ${ }^{104}$ M. Araujo, ${ }^{105}$ P. Bargassa, ${ }^{105}$ D. Bastos, ${ }^{105}$ A. Di Francesco, ${ }^{105}$ P. Faccioli, ${ }^{105}$ B. Galinhas, ${ }^{105}$ M. Gallinaro, ${ }^{105}$ J. Hollar, ${ }^{105}$ N. Leonardo, ${ }^{105}$ J. Seixas, ${ }^{105}$ K. Shchelina, ${ }^{105}$ G. Strong, ${ }^{105}$ O. Toldaiev, ${ }^{105}$ J. Varela, ${ }^{105}$ P. Bunin, ${ }^{106}$ M. Gavrilenko, ${ }^{106}$ 
A. Golunov, ${ }^{106}$ I. Golutvin, ${ }^{106}$ N. Gorbounov, ${ }^{106}$ I. Gorbunov, ${ }^{106}$ A. Kamenev, ${ }^{106}$ V. Karjavine, ${ }^{106}$ V. Korenkov, ${ }^{106}$ A. Lanev, ${ }^{106}$ A. Malakhov, ${ }^{106}$ V. Matveev, ${ }^{106, j j, k k}$ P. Moisenz, ${ }^{106}$ V. Palichik, ${ }^{106}$ V. Perelygin, ${ }^{106}$ M. Savina, ${ }^{106}$ S. Shmatov, ${ }^{106}$ N. Voytishin, ${ }^{106}$ B. S. Yuldashev, ${ }^{106,11}$ A. Zarubin, ${ }^{106}$ L. Chtchipounov, ${ }^{107}$ V. Golovtsov, ${ }^{107}$ Y. Ivanov, ${ }^{107}$ V. Kim, ${ }^{107, m m}$ E. Kuznetsova, ${ }^{107, n n}$ P. Levchenko, ${ }^{107}$ V. Murzin, ${ }^{107}$ V. Oreshkin, ${ }^{107}$ I. Smirnov, ${ }^{107}$ D. Sosnov, ${ }^{107}$ V. Sulimov, ${ }^{107}$ L. Uvarov, ${ }^{107}$ A. Vorobyev, ${ }^{107}$ Yu. Andreev, ${ }^{108}$ A. Dermenev, ${ }^{108}$ S. Gninenko, ${ }^{108}$ N. Golubev, ${ }^{108}$ A. Karneyeu, ${ }^{108}$ M. Kirsanov, ${ }^{108}$ N. Krasnikov, ${ }^{108}$ A. Pashenkov, ${ }^{108}$ D. Tlisov, ${ }^{108}$ A. Toropin, ${ }^{108}$ V. Epshteyn, ${ }^{109}$ V. Gavrilov,${ }^{109}$ N. Lychkovskaya, ${ }^{109}$ A. Nikitenko, ${ }^{109,00}$ V. Popov, ${ }^{109}$ I. Pozdnyakov, ${ }^{109}$ G. Safronov, ${ }^{109}$ A. Spiridonov, ${ }^{109}$ A. Stepennov, ${ }^{109}$ M. Toms, ${ }^{109}$ E. Vlasov, ${ }^{109}$ A. Zhokin, ${ }^{109}$ T. Aushev, ${ }^{110}$ O. Bychkova, ${ }^{111}$ R. Chistov, ${ }^{111, p p}$ M. Danilov, ${ }^{11, p p}$ S. Polikarpov, ${ }^{11, p p}$ E. Tarkovskii, ${ }^{111}$ V. Andreev, ${ }^{112}$ M. Azarkin, ${ }^{112}$ I. Dremin, ${ }^{112}$ M. Kirakosyan, ${ }^{112}$ A. Terkulov, ${ }^{112}$ A. Belyaev, ${ }^{113}$ E. Boos, ${ }^{113}$ M. Dubinin, ${ }^{11, q q}$ L. Dudko, ${ }^{113}$ A. Ershov, ${ }^{113}$ A. Gribushin, ${ }^{113}$ V. Klyukhin, ${ }^{113}$ O. Kodolova, ${ }^{113}$

I. Lokhtin, ${ }^{113}$ S. Obraztsov, ${ }^{113}$ S. Petrushanko, ${ }^{113}$ V. Savrin, ${ }^{113}$ A. Snigirev, ${ }^{113}$ A. Barnyakov, ${ }^{11, \text { rr }}$ V. Blinov, ${ }^{114, \mathrm{rr}}$ T. Dimova, ${ }^{114, \mathrm{rr}}$ L. Kardapoltsev, ${ }^{114, \mathrm{rr}}$ Y. Skovpen, ${ }^{114, \mathrm{rr}}$ I. Azhgirey, ${ }^{115}$ I. Bayshev, ${ }^{115}$ S. Bitioukov, ${ }^{115}$ V. Kachanov, ${ }^{115}$ D. Konstantinov, ${ }^{115}$ P. Mandrik, ${ }^{115}$ V. Petrov, ${ }^{115}$ R. Ryutin, ${ }^{115}$ S. Slabospitskii, ${ }^{115}$ A. Sobol, ${ }^{115}$ S. Troshin, ${ }^{115}$ N. Tyurin, ${ }^{115}$ A. Uzunian, ${ }^{115}$ A. Volkov, ${ }^{115}$ A. Babaev, ${ }^{116}$ A. Iuzhakov, ${ }^{116}$ V. Okhotnikov, ${ }^{116}$ V. Borchsh, ${ }^{117}$ V. Ivanchenko, ${ }^{117}$ E. Tcherniaev, ${ }^{117}$ P. Adzic, ${ }^{118, s s}$ P. Cirkovic, ${ }^{118}$ D. Devetak, ${ }^{118}$ M. Dordevic, ${ }^{118}$ P. Milenovic, ${ }^{118}$ J. Milosevic, ${ }^{118}$ M. Stojanovic, ${ }^{118}$ M. Aguilar-Benitez, ${ }^{119}$ J. Alcaraz Maestre, ${ }^{119}$ A. Álvarez Fernández, ${ }^{119}$ I. Bachiller, ${ }^{119}$ M. Barrio Luna, ${ }^{119}$ J. A. Brochero Cifuentes, ${ }^{119}$ C. A. Carrillo Montoya, ${ }^{119}$ M. Cepeda, ${ }^{119}$ M. Cerrada,${ }^{119}$ N. Colino, ${ }^{119}$ B. De La Cruz, ${ }^{119}$ A. Delgado Peris, ${ }^{119}$ C. Fernandez Bedoya,${ }^{119}$ J. P. Fernández Ramos, ${ }^{119}$ J. Flix ${ }^{119}$ M. C. Fouz, ${ }^{119}$ O. Gonzalez Lopez, ${ }^{119}$ S. Goy Lopez, ${ }^{119}$ J. M. Hernandez, ${ }^{119}$ M. I. Josa ${ }^{119}$ D. Moran, ${ }^{119}$ Á. Navarro Tobar, ${ }^{119}$ A. Pérez-Calero Yzquierdo, ${ }^{119}$ J. Puerta Pelayo, ${ }^{119}$ I. Redondo, ${ }^{119}$ L. Romero, ${ }^{119}$ S. Sánchez Navas, ${ }^{119}$ M. S. Soares, ${ }^{119}$ A. Triossi, ${ }^{119}$ C. Willmott,,${ }^{119}$ C. Albajar, ${ }^{120}$ J. F. de Trocóniz, ${ }^{120}$ B. Alvarez Gonzalez, ${ }^{121}$ J. Cuevas, ${ }^{121}$ C. Erice, ${ }^{121}$ J. Fernandez Menendez, ${ }^{121}$

S. Folgueras, ${ }^{121}$ I. Gonzalez Caballero, ${ }^{121}$ J. R. González Fernández, ${ }^{121}$ E. Palencia Cortezon, ${ }^{121}$ V. Rodríguez Bouza, ${ }^{121}$ S. Sanchez Cruz, ${ }^{121}$ I. J. Cabrillo, ${ }^{122}$ A. Calderon, ${ }^{122}$ B. Chazin Quero, ${ }^{122}$ J. Duarte Campderros, ${ }^{122}$ M. Fernandez, ${ }^{122}$ P. J. Fernández Manteca, ${ }^{122}$ A. García Alonso, ${ }^{122}$ G. Gomez,${ }^{122}$ C. Martinez Rivero, ${ }^{122}$ P. Martinez Ruiz del Arbol, ${ }^{122}$ F. Matorras, ${ }^{122}$ J. Piedra Gomez, ${ }^{122}$ C. Prieels, ${ }^{122}$ T. Rodrigo, ${ }^{122}$ A. Ruiz-Jimeno, ${ }^{122}$ L. Russo, ${ }^{122, t \mathrm{t}}$ L. Scodellaro, ${ }^{122}$ N. Trevisani, ${ }^{122}$ I. Vila, ${ }^{122}$ J. M. Vizan Garcia, ${ }^{122}$ K. Malagalage, ${ }^{123}$ W. G. D. Dharmaratna, ${ }^{124}$ N. Wickramage, ${ }^{124}$ D. Abbaneo, ${ }^{125}$ B. Akgun, ${ }^{125}$ E. Auffray,${ }^{125}$ G. Auzinger, ${ }^{125}$ J. Baechler, ${ }^{125}$ P. Baillon, ${ }^{125}$ A. H. Ball, ${ }^{125}$ D. Barney, ${ }^{125}$ J. Bendavid, ${ }^{125}$ M. Bianco, ${ }^{125}$ A. Bocci, ${ }^{125}$ E. Bossini, ${ }^{125}$ C. Botta, ${ }^{125}$ E. Brondolin, ${ }^{125}$ T. Camporesi, ${ }^{125}$ A. Caratelli, ${ }^{125}$ G. Cerminara, ${ }^{125}$ E. Chapon, ${ }^{125}$ G. Cucciati, ${ }^{125}$ D. d'Enterria, ${ }^{125}$ A. Dabrowski, ${ }^{125}$ N. Daci, ${ }^{125}$ V. Daponte, ${ }^{125}$ A. David, ${ }^{125}$ O. Davignon, ${ }^{125}$ A. De Roeck, ${ }^{125}$ N. Deelen, ${ }^{125}$ M. Deile, ${ }^{125}$ M. Dobson, ${ }^{125}$ M. Dünser ${ }^{125}$ N. Dupont, ${ }^{125}$ A. Elliott-Peisert, ${ }^{125}$ F. Fallavollita, ${ }^{125, \text { uu }}$ D. Fasanella, ${ }^{125}$ G. Franzoni, ${ }^{125}$ J. Fulcher, ${ }^{125}$ W. Funk, ${ }^{125}$ S. Giani, ${ }^{125}$ D. Gigi, ${ }^{125}$ A. Gilbert ${ }^{125}$ K. Gill, ${ }^{125}$ F. Glege, ${ }^{125}$ M. Gruchala, ${ }^{125}$ M. Guilbaud, ${ }^{125}$ D. Gulhan, ${ }^{125}$ J. Hegeman, ${ }^{125}$ C. Heidegger, ${ }^{125}$ Y. Iiyama, ${ }^{125}$ V. Innocente, ${ }^{125}$ P. Janot, ${ }^{125}$ O. Karacheban, ${ }^{125, t}$ J. Kaspar, ${ }^{125}$ J. Kieseler, ${ }^{125}$ M. Krammer, ${ }^{125, b}$ C. Lange, ${ }^{125}$ P. Lecoq, ${ }^{125}$ C. Lourenço, ${ }^{125}$ L. Malgeri, ${ }^{125}$ M. Mannelli, ${ }^{125}$ A. Massironi, ${ }^{125}$ F. Meijers, ${ }^{125}$ J. A. Merlin, ${ }^{125}$ S. Mersi, ${ }^{125}$ E. Meschi, ${ }^{125}$ F. Moortgat, ${ }^{125}$ M. Mulders, ${ }^{125}$ J. Ngadiuba, ${ }^{125}$ S. Nourbakhsh, ${ }^{125}$ S. Orfanelli, ${ }^{125}$ L. Orsini, ${ }^{125}$ F. Pantaleo, ${ }^{125, q}$ L. Pape, ${ }^{125}$ E. Perez, ${ }^{125}$ M. Peruzzi, ${ }^{125}$ A. Petrilli, ${ }^{125}$ G. Petrucciani, ${ }^{125}$ A. Pfeiffer, ${ }^{125}$ M. Pierini, ${ }^{125}$ F. M. Pitters, ${ }^{125}$ D. Rabady, ${ }^{125}$ A. Racz, ${ }^{125}$ M. Rovere, ${ }^{125}$ H. Sakulin, ${ }^{125}$ C. Schäfer, ${ }^{125}$ C. Schwick, ${ }^{125}$ M. Selvaggi, ${ }^{125}$ A. Sharma,${ }^{125}$ P. Silva, ${ }^{125}$ W. Snoeys, ${ }^{125}$ P. Sphicas, ${ }^{125, \mathrm{vv}}$ J. Steggemann, ${ }^{125}$ V. R. Tavolaro, ${ }^{125}$ D. Treille, ${ }^{125}$ A. Tsirou, ${ }^{125}$ A. Vartak, ${ }^{125}$ M. Verzetti, ${ }^{125}$ W. D. Zeuner, ${ }^{125}$ L. Caminada, ${ }^{126, w w}$ K. Deiters, ${ }^{126}$ W. Erdmann, ${ }^{126}$ R. Horisberger, ${ }^{126}$ Q. Ingram, ${ }^{126}$ H. C. Kaestli, ${ }^{126}$ D. Kotlinski, ${ }^{126}$ U. Langenegger ${ }^{126}$ T. Rohe, ${ }^{126}$ S. A. Wiederkehr, ${ }^{126}$ M. Backhaus, ${ }^{127}$ P. Berger, ${ }^{127}$ N. Chernyavskaya, ${ }^{127}$ G. Dissertori, ${ }^{127}$ M. Dittmar, ${ }^{127}$ M. Donegà, ${ }^{127}$ C. Dorfer,${ }^{127}$ T. A. Gómez Espinosa, ${ }^{127}$ C. Grab, ${ }^{127}$ D. Hits, ${ }^{127}$ T. Klijnsma, ${ }^{127}$ W. Lustermann, ${ }^{127}$ R. A. Manzoni, ${ }^{127}$ M. Marionneau, ${ }^{127}$ M. T. Meinhard, ${ }^{127}$ F. Micheli, ${ }^{127}$ P. Musella, ${ }^{127}$ F. Nessi-Tedaldi, ${ }^{127}$ F. Pauss, ${ }^{127}$ G. Perrin, ${ }^{127}$ L. Perrozzi, ${ }^{127}$ S. Pigazzini, ${ }^{127}$ M. Reichmann, ${ }^{127}$ C. Reissel, ${ }^{127}$ T. Reitenspiess, ${ }^{127}$ D. Ruini, ${ }^{127}$ D. A. Sanz Becerra, ${ }^{127}$ M. Schönenberger, ${ }^{127}$ L. Shchutska, ${ }^{127}$ M. L. Vesterbacka Olsson, ${ }^{127}$ R. Wallny, ${ }^{127}$ D. H. Zhu, ${ }^{127}$ T. K. Aarrestad, ${ }^{128}$ C. Amsler, ${ }^{128, x x}$ D. Brzhechko, ${ }^{128}$ M. F. Canelli, ${ }^{128}$ A. De Cosa, ${ }^{128}$ R. Del Burgo, ${ }^{128}$ S. Donato, ${ }^{128}$ B. Kilminster, ${ }^{128}$ S. Leontsinis, ${ }^{128}$ V. M. Mikuni, ${ }^{128}$ I. Neutelings, ${ }^{128}$ G. Rauco, ${ }^{128}$ P. Robmann, ${ }^{128}$ D. Salerno, ${ }^{128}$ K. Schweiger, ${ }^{128}$ C. Seitz, ${ }^{128}$ Y. Takahashi, ${ }^{128}$ S. Wertz, ${ }^{128}$ A. Zucchetta, ${ }^{128}$ T. H. Doan, ${ }^{129}$ C. M. Kuo, ${ }^{129}$ W. Lin, ${ }^{129}$ A. Roy, ${ }^{129}$ S. S. Yu, ${ }^{129}$ P. Chang, ${ }^{130}$ Y. Chao, ${ }^{130}$ K. F. Chen, ${ }^{130}$ P. H. Chen, ${ }^{130}$ W.-S. Hou ${ }^{130}$ Y. y. Li, ${ }^{130}$ R.-S. Lu, ${ }^{130}$ E. Paganis, ${ }^{130}$ A. Psallidas, ${ }^{130}$ A. Steen, ${ }^{130}$ B. Asavapibhop, ${ }^{131}$ C. Asawatangtrakuldee, ${ }^{131}$ 
N. Srimanobhas, ${ }^{131}$ N. Suwonjandee, ${ }^{131}$ A. Bat, ${ }^{132}$ F. Boran, ${ }^{132}$ S. Cerci, ${ }^{132, y y}$ S. Damarseckin, ${ }^{132, z z}$ Z. S. Demiroglu, ${ }^{132}$ F. Dolek, ${ }^{132}$ C. Dozen, ${ }^{132}$ I. Dumanoglu, ${ }^{132}$ G. Gokbulut, ${ }^{132}$ E. Gurpinar Guler, ${ }^{132, \text { aaa }}$ Y. Guler, ${ }^{132}$ I. Hos, ${ }^{132, \text { bbb }}$ C. Isik, ${ }^{132}$ E. E. Kangal, ${ }^{132, \text { ccc }}$ O. Kara, ${ }^{132}$ A. Kayis Topaksu, ${ }^{132}$ U. Kiminsu, ${ }^{132}$ M. Oglakci, ${ }^{132}$ G. Onengut, ${ }^{132}$ K. Ozdemir, ${ }^{132, d d d}$ S. Ozturk, ${ }^{132, \text { eee }}$ A. E. Simsek, ${ }^{132}$ D. Sunar Cerci, ${ }^{132, y y}$ U. G. Tok, ${ }^{132}$ S. Turkcapar, ${ }^{132}$ I. S. Zorbakir, ${ }^{132}$ C. Zorbilmez, ${ }^{132}$ B. Isildak, ${ }^{133, \text { fff }}$ G. Karapinar, ${ }^{133, g g g}$ M. Yalvac, ${ }^{133}$ I. O. Atakisi, ${ }^{134}$ E. Gülmez, ${ }^{134}$ M. Kaya, ${ }^{134, \text { hhh }}$ O. Kaya, ${ }^{134, \text { iii }}$ B. Kaynak, ${ }^{134}$ Ö. Özçelik, ${ }^{134}$ S. Tekten, ${ }^{134}$ E. A. Yetkin, ${ }^{134, j j j}$ A. Cakir, ${ }^{135}$ Y. Komurcu, ${ }^{135}$ S. Sen, ${ }^{135, k k k}$ S. Ozkorucuklu, ${ }^{136}$ B. Grynyov, ${ }^{137}$ L. Levchuk, ${ }^{138}$ F. Ball, ${ }^{139}$ E. Bhal,${ }^{139}$ S. Bologna, ${ }^{139}$ J. J. Brooke, ${ }^{139}$ D. Burns,${ }^{139}$ E. Clement, ${ }^{139}$ D. Cussans, ${ }^{139}$ H. Flacher, ${ }^{139}$ J. Goldstein, ${ }^{139}$ G. P. Heath, ${ }^{139}$ H. F. Heath, ${ }^{139}$ L. Kreczko, ${ }^{139}$ S. Paramesvaran, ${ }^{139}$ B. Penning, ${ }^{139}$ T. Sakuma,${ }^{139}$ S. Seif El Nasr-Storey, ${ }^{139}$ D. Smith, ${ }^{139}$ V. J. Smith, ${ }^{139}$ J. Taylor, ${ }^{139}$ A. Titterton, ${ }^{139}$ K. W. Bell, ${ }^{140}$ A. Belyaev, ${ }^{140,111}$ C. Brew, ${ }^{140}$ R. M. Brown, ${ }^{140}$ D. Cieri, ${ }^{140}$ D. J. A. Cockerill, ${ }^{140}$ J. A. Coughlan, ${ }^{140}$ K. Harder, ${ }^{140}$ S. Harper,${ }^{140}$ J. Linacre, ${ }^{140}$ K. Manolopoulos, ${ }^{140}$ D. M. Newbold,${ }^{140}$ E. Olaiya, ${ }^{140}$ D. Petyt, ${ }^{140}$ T. Reis,${ }^{140}$ T. Schuh, ${ }^{140}$

C. H. Shepherd-Themistocleous, ${ }^{140}$ A. Thea ${ }^{140}$ I. R. Tomalin, ${ }^{140}$ T. Williams, ${ }^{140}$ W. J. Womersley,${ }^{140}$ R. Bainbridge,${ }^{141}$ P. Bloch ${ }^{141}$ J. Borg, ${ }^{141}$ S. Breeze, ${ }^{141}$ O. Buchmuller, ${ }^{141}$ A. Bundock,${ }^{141}$ Gurpreet Singh Chahal, ${ }^{141, \mathrm{mmm}}$ D. Colling, ${ }^{141}$ P. Dauncey, ${ }^{141}$ G. Davies, ${ }^{141}$ M. Della Negra, ${ }^{141}$ R. Di Maria,${ }^{141}$ P. Everaerts, ${ }^{141}$ G. Hall,,${ }^{141}$ G. Iles, ${ }^{141}$ T. James, ${ }^{141}$ M. Komm, ${ }^{141}$ C. Laner, ${ }^{141}$ L. Lyons, ${ }^{141}$ A.-M. Magnan, ${ }^{141}$ S. Malik, ${ }^{141}$ A. Martelli, ${ }^{141}$ V. Milosevic, ${ }^{141}$ J. Nash, ${ }^{141, n n n}$ V. Palladino, ${ }^{141}$ M. Pesaresi, ${ }^{141}$ D. M. Raymond, ${ }^{141}$ A. Richards, ${ }^{141}$ A. Rose,${ }^{141}$ E. Scott, ${ }^{141}$ C. Seez, ${ }^{141}$ A. Shtipliyski, ${ }^{141}$ M. Stoye,${ }^{141}$ T. Strebler ${ }^{141}$ S. Summers, ${ }^{141}$ A. Tapper, ${ }^{141}$ K. Uchida, ${ }^{141}$ T. Virdee,${ }^{141, q}$ N. Wardle, ${ }^{141}$ D. Winterbottom, ${ }^{141}$ J. Wright, ${ }^{141}$ A. G. Zecchinelli, ${ }^{141}$ S. C. Zenz, ${ }^{141}$ J. E. Cole,${ }^{142}$ P. R. Hobson, ${ }^{142}$ A. Khan,${ }^{142}$ P. Kyberd, ${ }^{142}$ C. K. Mackay, ${ }^{142}$ A. Morton, ${ }^{142}$ I. D. Reid, ${ }^{142}$ L. Teodorescu, ${ }^{142}$ S. Zahid, ${ }^{142}$ K. Call, ${ }^{143}$ J. Dittmann, ${ }^{143}$ K. Hatakeyama, ${ }^{143}$ C. Madrid,${ }^{143}$ B. McMaster, ${ }^{143}$ N. Pastika, ${ }^{143}$ C. Smith,${ }^{143}$ R. Bartek, ${ }^{144}$ A. Dominguez, ${ }^{144}$ R. Uniyal, ${ }^{144}$ A. Buccilli, ${ }^{145}$ S. I. Cooper, ${ }^{145}$ C. Henderson, ${ }^{145}$ P. Rumerio, ${ }^{145}$ C. West,${ }^{145}$ D. Arcaro, ${ }^{146}$ T. Bose, ${ }^{146}$ Z. Demiragli, ${ }^{146}$ D. Gastler,${ }^{146}$ S. Girgis,${ }^{146}$ D. Pinna, ${ }^{146}$ C. Richardson, ${ }^{146}$ J. Rohlf, ${ }^{146}$ D. Sperka, ${ }^{146}$ I. Suarez, ${ }^{146}$ L. Sulak ${ }^{146}$ D. Zou, ${ }^{146}$ G. Benelli, ${ }^{147}$ B. Burkle, ${ }^{147}$ X. Coubez ${ }^{147}$ D. Cutts, ${ }^{147}$ Y. t. Duh ${ }^{147}$ M. Hadley, ${ }^{147}$ J. Hakala, ${ }^{147}$ U. Heintz, ${ }^{147}$ J. M. Hogan,${ }^{147,000}$ K. H. M. Kwok, ${ }^{147}$ E. Laird, ${ }^{147}$ G. Landsberg, ${ }^{147}$ J. Lee, ${ }^{147}$ Z. Mao,${ }^{147}$ M. Narain, ${ }^{147}$ S. Sagir, ${ }^{147, p p p}$ R. Syarif, ${ }^{147}$ E. Usai, ${ }^{147}$ D. Yu, ${ }^{147}$ R. Band, ${ }^{148}$ C. Brainerd, ${ }^{148}$ R. Breedon, ${ }^{148}$ M. Calderon De La Barca Sanchez, ${ }^{148}$ M. Chertok, ${ }^{148}$ J. Conway, ${ }^{148}$ R. Conway, ${ }^{148}$ P. T. Cox, ${ }^{148}$ R. Erbacher, ${ }^{148}$ C. Flores, ${ }^{148}$ G. Funk, ${ }^{148}$ F. Jensen, ${ }^{148}$ W. Ko, ${ }^{148}$ O. Kukral, ${ }^{148}$ R. Lander, ${ }^{148}$ M. Mulhearn, ${ }^{148}$ D. Pellett, ${ }^{148}$ J. Pilot, ${ }^{148}$ M. Shi, ${ }^{148}$ D. Stolp, ${ }^{148}$ D. Taylor, ${ }^{148}$ K. Tos, ${ }^{148}$ M. Tripathi, ${ }^{148}$ Z. Wang, ${ }^{148}$ F. Zhang, ${ }^{148}$ M. Bachtis, ${ }^{149}$ C. Bravo, ${ }^{149}$ R. Cousins, ${ }^{149}$ A. Dasgupta ${ }^{149}$ A. Florent,${ }^{149}$ J. Hauser, ${ }^{149}$ M. Ignatenko, ${ }^{149}$ N. Mccoll ${ }^{149}$ W. A. Nash, ${ }^{149}$ S. Regnard, ${ }^{149}$ D. Saltzberg, ${ }^{149}$ C. Schnaible, ${ }^{149}$ B. Stone, ${ }^{149}$ V. Valuev, ${ }^{149}$ K. Burt, ${ }^{150}$ R. Clare, ${ }^{150}$ J. W. Gary, ${ }^{150}$ S. M. A. Ghiasi Shirazi, ${ }^{150}$ G. Hanson, ${ }^{150}$ G. Karapostoli, ${ }^{150}$ E. Kennedy, ${ }^{150}$ O. R. Long, ${ }^{150}$

M. Olmedo Negrete, ${ }^{150}$ M. I. Paneva, ${ }^{150}$ W. Si, ${ }^{150}$ L. Wang, ${ }^{150}$ H. Wei, ${ }^{150}$ S. Wimpenny, ${ }^{150}$ B. R. Yates, ${ }^{150}$ Y. Zhang, ${ }^{150}$ J. G. Branson, ${ }^{151}$ P. Chang, ${ }^{151}$ S. Cittolin, ${ }^{151}$ M. Derdzinski, ${ }^{151}$ R. Gerosa, ${ }^{151}$ D. Gilbert ${ }^{151}$ B. Hashemi, ${ }^{151}$ D. Klein, ${ }^{151}$ V. Krutelyov, ${ }^{151}$ J. Letts, ${ }^{151}$ M. Masciovecchio, ${ }^{151}$ S. May, ${ }^{151}$ S. Padhi ${ }^{151}$ M. Pieri, ${ }^{151}$ V. Sharma,${ }^{151}$ M. Tadel, ${ }^{151}$ F. Würthwein, ${ }^{151}$ A. Yagil, ${ }^{151}$ G. Zevi Della Porta, ${ }^{151}$ N. Amin, ${ }^{152}$ R. Bhandari, ${ }^{152}$ C. Campagnari, ${ }^{152}$ M. Citron, ${ }^{152}$ V. Dutta, ${ }^{152}$ M. Franco Sevilla, ${ }^{152}$ L. Gouskos, ${ }^{152}$ J. Incandela, ${ }^{152}$ B. Marsh ${ }^{152}$ H. Mei, ${ }^{152}$ A. Ovcharova,${ }^{152}$ H. Qu, ${ }^{152}$ J. Richman, ${ }^{152}$ U. Sarica, ${ }^{152}$ D. Stuart, ${ }^{152}$ S. Wang, ${ }^{152}$ J. Yoo, ${ }^{152}$ D. Anderson, ${ }^{153}$ A. Bornheim, ${ }^{153}$ O. Cerri, ${ }^{153}$ I. Dutta, ${ }^{153}$ J. M. Lawhorn, ${ }^{153}$ N. Lu, ${ }^{153}$ J. Mao, ${ }^{153}$ H. B. Newman, ${ }^{153}$ T. Q. Nguyen, ${ }^{153}$ J. Pata, ${ }^{153}$ M. Spiropulu, ${ }^{153}$ J. R. Vlimant, ${ }^{153}$ S. Xie, ${ }^{153}$ Z. Zhang, ${ }^{153}$ R. Y. Zhu, ${ }^{153}$ M. B. Andrews, ${ }^{154}$ T. Ferguson, ${ }^{154}$ T. Mudholkar, ${ }^{154}$ M. Paulini, ${ }^{154}$ M. Sun, ${ }^{154}$ I. Vorobiev, ${ }^{154}$ M. Weinberg, ${ }^{154}$ J. P. Cumalat, ${ }^{155}$ W. T. Ford, ${ }^{155}$ A. Johnson, ${ }^{155}$ E. MacDonald, ${ }^{155}$ T. Mulholland, ${ }^{155}$ R. Patel, ${ }^{155}$ A. Perloff, ${ }^{155}$ K. Stenson, ${ }^{155}$ K. A. Ulmer, ${ }^{155}$ S. R. Wagner ${ }^{155}$ J. Alexander, ${ }^{156}$ J. Chaves,${ }^{156}$ Y. Cheng, ${ }^{156}$ J. Chu, ${ }^{156}$ A. Datta, ${ }^{156}$ A. Frankenthal, ${ }^{156}$ K. Mcdermott, ${ }^{156}$ N. Mirman, ${ }^{156}$ J. R. Patterson, ${ }^{156}$ D. Quach, ${ }^{156}$ A. Rinkevicius, ${ }^{156, \text { qqq }}$ A. Ryd, ${ }^{156}$ S. M. Tan, ${ }^{156}$ Z. Tao, ${ }^{156}$ J. Thom, ${ }^{156}$ P. Wittich, ${ }^{156}$ M. Zientek, ${ }^{156}$ S. Abdullin, ${ }^{157}$ M. Albrow, ${ }^{157}$ M. Alyari, ${ }^{157}$ G. Apollinari, ${ }^{157}$ A. Apresyan, ${ }^{157}$ A. Apyan, ${ }^{157}$ S. Banerjee, ${ }^{157}$ L. A. T. Bauerdick,${ }^{157}$ A. Beretvas, ${ }^{157}$ J. Berryhill, ${ }^{157}$ P. C. Bhat,${ }^{157}$ K. Burkett, ${ }^{157}$ J. N. Butler, ${ }^{157}$ A. Canepa, ${ }^{157}$ G. B. Cerati, ${ }^{157}$ H. W. K. Cheung, ${ }^{157}$ F. Chlebana, ${ }^{157}$ M. Cremonesi,${ }^{157}$ J. Duarte, ${ }^{157}$ V. D. Elvira, ${ }^{157}$ J. Freeman, ${ }^{157}$ Z. Gecse, ${ }^{157}$ E. Gottschalk, ${ }^{157}$ L. Gray, ${ }^{157}$ D. Green, ${ }^{157}$ S. Grünendahl,${ }^{157}$ O. Gutsche,${ }^{157}$ Allison Reinsvold Hall, ${ }^{157}$ J. Hanlon, ${ }^{157}$ R. M. Harris ${ }^{157}$ S. Hasegawa, ${ }^{157}$ R. Heller, ${ }^{157}$ J. Hirschauer ${ }^{157}$ B. Jayatilaka, ${ }^{157}$ S. Jindariani, ${ }^{157}$ M. Johnson, ${ }^{157}$ U. Joshi, ${ }^{157}$ B. Klima, ${ }^{157}$ M. J. Kortelainen, ${ }^{157}$ B. Kreis, ${ }^{157}$ S. Lammel,${ }^{157}$ J. Lewis, ${ }^{157}$ D. Lincoln, ${ }^{157}$ R. Lipton, ${ }^{157}$ M. Liu ${ }^{157}$ T. Liu, ${ }^{157}$ J. Lykken, ${ }^{157}$ K. Maeshima, ${ }^{157}$ J. M. Marraffino, ${ }^{157}$ D. Mason, ${ }^{157}$ P. McBride,${ }^{157}$ P. Merkel, ${ }^{157}$ S. Mrenna,${ }^{157}$ S. Nahn, ${ }^{157}$ 
V. O’Dell, ${ }^{157}$ V. Papadimitriou, ${ }^{157}$ K. Pedro, ${ }^{157}$ C. Pena, ${ }^{157}$ G. Rakness, ${ }^{157}$ F. Ravera, ${ }^{157}$ L. Ristori, ${ }^{157}$ B. Schneider, ${ }^{157}$ E. Sexton-Kennedy, ${ }^{157}$ N. Smith, ${ }^{157}$ A. Soha, ${ }^{157}$ W. J. Spalding, ${ }^{157}$ L. Spiegel, ${ }^{157}$ S. Stoynev ${ }^{157}$ J. Strait, ${ }^{157}$ N. Strobbe, ${ }^{157}$ L. Taylor, ${ }^{157}$ S. Tkaczyk, ${ }^{157}$ N. V. Tran, ${ }^{157}$ L. Uplegger, ${ }^{157}$ E. W. Vaandering, ${ }^{157}$ C. Vernieri, ${ }^{157}$ M. Verzocchi,${ }^{157}$ R. Vidal,,${ }^{157}$ M. Wang, ${ }^{157}$ H. A. Weber, ${ }^{157}$ D. Acosta, ${ }^{158}$ P. Avery, ${ }^{158}$ P. Bortignon, ${ }^{158}$ D. Bourilkov, ${ }^{158}$ A. Brinkerhoff, ${ }^{158}$ L. Cadamuro, ${ }^{158}$ A. Carnes, ${ }^{158}$ V. Cherepanov, ${ }^{158}$ D. Curry, ${ }^{158}$ F. Errico, ${ }^{158}$ R. D. Field, ${ }^{158}$ S. V. Gleyzer, ${ }^{158}$ B. M. Joshi, ${ }^{158}$ M. Kim, ${ }^{158}$ J. Konigsberg, ${ }^{158}$ A. Korytov, ${ }^{158}$ K. H. Lo,${ }^{158}$ P. Ma ${ }^{158}$ K. Matchev,${ }^{158}$ N. Menendez, ${ }^{158}$ G. Mitselmakher, ${ }^{158}$ D. Rosenzweig, ${ }^{158}$ K. Shi, ${ }^{158}$ J. Wang, ${ }^{158}$ S. Wang, ${ }^{158}$ X. Zuo, ${ }^{158}$ Y. R. Joshi ${ }^{159}$ T. Adams, ${ }^{160}$ A. Askew, ${ }^{160}$ S. Hagopian, ${ }^{160}$ V. Hagopian, ${ }^{160}$ K. F. Johnson, ${ }^{160}$ R. Khurana, ${ }^{160}$ T. Kolberg, ${ }^{160}$ G. Martinez, ${ }^{160}$ T. Perry, ${ }^{160}$ H. Prosper, ${ }^{160}$ C. Schiber, ${ }^{160}$ R. Yohay, ${ }^{160}$ J. Zhang, ${ }^{160}$ M. M. Baarmand, ${ }^{161}$ V. Bhopatkar, ${ }^{161}$ M. Hohlmann, ${ }^{161}$ D. Noonan, ${ }^{161}$ M. Rahmani, ${ }^{161}$ M. Saunders, ${ }^{161}$ F. Yumiceva, ${ }^{161}$ M. R. Adams, ${ }^{162}$ L. Apanasevich, ${ }^{162}$ D. Berry, ${ }^{162}$ R. R. Betts, ${ }^{162}$ R. Cavanaugh, ${ }^{162}$ X. Chen, ${ }^{162}$ S. Dittmer, ${ }^{162}$ O. Evdokimov, ${ }^{162}$ C. E. Gerber, ${ }^{162}$ D. A. Hangal, ${ }^{162}$ D. J. Hofman, ${ }^{162}$ K. Jung, ${ }^{162}$ C. Mills, ${ }^{162}$ T. Roy, ${ }^{162}$ M. B. Tonjes, ${ }^{162}$ N. Varelas, ${ }^{162}$ H. Wang, ${ }^{162}$ X. Wang, ${ }^{162} \mathrm{Z}$. Wu, ${ }^{162}$ M. Alhusseini, ${ }^{163}$ B. Bilki, ${ }^{163, \text { aaa }}$ W. Clarida, ${ }^{163}$ K. Dilsiz, ${ }^{163, \text { rrr }}$ S. Durgut, ${ }^{163}$ R. P. Gandrajula, ${ }^{163}$ M. Haytmyradov, ${ }^{163}$ V. Khristenko, ${ }^{163}$ O. K. Köseyan, ${ }^{163}$ J.-P. Merlo, ${ }^{163}$ A. Mestvirishvili, ${ }^{163, \text { sss }}$ A. Moeller, ${ }^{163}$ J. Nachtman, ${ }^{163}$ H. Ogul, ${ }^{163, \text { tt }}$ Y. Onel,${ }^{163}$ F. Ozok, ${ }^{163, \text { uuu }}$ A. Penzo, ${ }^{163}$ C. Snyder, ${ }^{163}$ E. Tiras, ${ }^{163}$ J. Wetzel, ${ }^{163}$ B. Blumenfeld, ${ }^{164}$ A. Cocoros, ${ }^{164}$ N. Eminizer, ${ }^{164}$ D. Fehling, ${ }^{164}$ L. Feng, ${ }^{164}$ A. V. Gritsan, ${ }^{164}$ W. T. Hung, ${ }^{164}$ P. Maksimovic, ${ }^{164}$ J. Roskes, ${ }^{164}$ M. Swartz, ${ }^{164}$ M. Xiao, ${ }^{164}$ C. Baldenegro Barrera, ${ }^{165}$ P. Baringer, ${ }^{165}$

A. Bean, ${ }^{165}$ S. Boren, ${ }^{165}$ J. Bowen, ${ }^{165}$ A. Bylinkin, ${ }^{165}$ T. Isidori, ${ }^{165}$ S. Khalil, ${ }^{165}$ J. King,${ }^{165}$ G. Krintiras, ${ }^{165}$

A. Kropivnitskaya, ${ }^{165}$ C. Lindsey, ${ }^{165}$ D. Majumder, ${ }^{165}$ W. Mcbrayer, ${ }^{165}$ N. Minafra ${ }^{165}$ M. Murray, ${ }^{165}$ C. Rogan, ${ }^{165}$ C. Royon, ${ }^{165}$ S. Sanders, ${ }^{165}$ E. Schmitz, ${ }^{165}$ J. D. Tapia Takaki, ${ }^{165}$ Q. Wang, ${ }^{165}$ J. Williams, ${ }^{165}$ G. Wilson, ${ }^{165}$ S. Duric, ${ }^{166}$ A. Ivanov, ${ }^{166}$ K. Kaadze, ${ }^{166}$ D. Kim, ${ }^{166}$ Y. Maravin, ${ }^{166}$ D. R. Mendis, ${ }^{166}$ T. Mitchell, ${ }^{166}$ A. Modak, ${ }^{166}$ A. Mohammadi, ${ }^{166}$ F. Rebassoo, ${ }^{167}$ D. Wright, ${ }^{167}$ A. Baden, ${ }^{168}$ O. Baron, ${ }^{168}$ A. Belloni, ${ }^{168}$ S. C. Eno, ${ }^{168}$ Y. Feng, ${ }^{168}$ N. J. Hadley, ${ }^{168}$ S. Jabeen, ${ }^{168}$ G. Y. Jeng, ${ }^{168}$ R. G. Kellogg, ${ }^{168}$ J. Kunkle, ${ }^{168}$ A. C. Mignerey ${ }^{168}$ S. Nabili, ${ }^{168}$ F. Ricci-Tam, ${ }^{168}$ M. Seidel, ${ }^{168}$ Y. H. Shin, ${ }^{168}$ A. Skuja, ${ }^{168}$ S. C. Tonwar, ${ }^{168}$ K. Wong, ${ }^{168}$ D. Abercrombie, ${ }^{169}$ B. Allen, ${ }^{169}$ A. Baty, ${ }^{169}$ R. Bi, ${ }^{169}$ S. Brandt, ${ }^{169}$ W. Busza, ${ }^{169}$ I. A. Cali, ${ }^{169}$ M. D'Alfonso, ${ }^{169}$ G. Gomez Ceballos, ${ }^{169}$ M. Goncharov ${ }^{169}$ P. Harris, ${ }^{169}$ D. Hsu, ${ }^{169}$ M. Hu, ${ }^{169}$ M. Klute, ${ }^{169}$ D. Kovalskyi, ${ }^{169}$ Y.-J. Lee, ${ }^{169}$ P. D. Luckey, ${ }^{169}$ B. Maier, ${ }^{169}$ A. C. Marini, ${ }^{169}$ C. Mcginn, ${ }^{169}$ C. Mironov, ${ }^{169}$ S. Narayanan, ${ }^{169}$ X. Niu, ${ }^{169}$ C. Paus, ${ }^{169}$ D. Rankin, ${ }^{169}$ C. Roland, ${ }^{169}$ G. Roland, ${ }^{169}$ Z. Shi, ${ }^{169}$ G. S. F. Stephans, ${ }^{169}$ K. Sumorok, ${ }^{169}$ K. Tatar, ${ }^{169}$ D. Velicanu, ${ }^{169}$ J. Wang, ${ }^{169}$ T. W. Wang, ${ }^{169}$ B. Wyslouch, ${ }^{169}$ A. C. Benvenuti, ${ }^{170, a}$ R. M. Chatterjee, ${ }^{170}$ A. Evans, ${ }^{170}$ S. Guts, ${ }^{170}$ P. Hansen, ${ }^{170}$ J. Hiltbrand, ${ }^{170}$ S. Kalafut, ${ }^{170}$ Y. Kubota,${ }^{170}$ Z. Lesko, ${ }^{170}$ J. Mans,${ }^{170}$ R. Rusack, ${ }^{170}$ M. A. Wadud, ${ }^{170}$ J. G. Acosta, ${ }^{171}$ S. Oliveros, ${ }^{171}$ K. Bloom, ${ }^{172}$ D. R. Claes, ${ }^{172}$ C. Fangmeier, ${ }^{172}$ L. Finco,${ }^{172}$ F. Golf,,${ }^{172}$ R. Gonzalez Suarez, ${ }^{172}$ R. Kamalieddin, ${ }^{172}$ I. Kravchenko, ${ }^{172}$ J. E. Siado, ${ }^{172}$ G. R. Snow, ${ }^{172}$ B. Stieger, ${ }^{172}$ G. Agarwal,${ }^{173}$ C. Harrington, ${ }^{173}$ I. Iashvili, ${ }^{173}$ A. Kharchilava, ${ }^{173}$ C. Mclean, ${ }^{173}$ D. Nguyen, ${ }^{173}$ A. Parker, ${ }^{173}$ J. Pekkanen, ${ }^{173}$ S. Rappoccio, ${ }^{173}$ B. Roozbahani, ${ }^{173}$ G. Alverson, ${ }^{174}$ E. Barberis,${ }^{174}$ C. Freer, ${ }^{174}$ Y. Haddad,${ }^{174}$ A. Hortiangtham,${ }^{174}$ G. Madigan, ${ }^{174}$ D. M. Morse, ${ }^{174}$ T. Orimoto, ${ }^{174}$ L. Skinnari, ${ }^{174}$ A. Tishelman-Charny, ${ }^{174}$ T. Wamorkar, ${ }^{174}$ B. Wang, ${ }^{174}$ A. Wisecarver, ${ }^{174}$ D. Wood, ${ }^{174}$ S. Bhattacharya, ${ }^{175}$ J. Bueghly, ${ }^{175}$ T. Gunter, ${ }^{175}$ K. A. Hahn,,${ }^{175}$ N. Odell, ${ }^{175}$ M. H. Schmitt, ${ }^{175}$ K. Sung, ${ }^{175}$ M. Trovato, ${ }^{175}$ M. Velasco, ${ }^{175}$ R. Bucci, ${ }^{176}$ N. Dev, ${ }^{176}$ R. Goldouzian, ${ }^{176}$ M. Hildreth, ${ }^{176}$ K. Hurtado Anampa, ${ }^{176}$ C. Jessop, ${ }^{176}$ D. J. Karmgard, ${ }^{176}$ K. Lannon, ${ }^{176}$ W. Li,${ }^{176}$ N. Loukas, ${ }^{176}$ N. Marinelli, ${ }^{176}$ I. Mcalister, ${ }^{176}$ F. Meng, ${ }^{176}$ C. Mueller, ${ }^{176}$ Y. Musienko, ${ }^{176, j j}$ M. Planer, ${ }^{176}$ R. Ruchti, ${ }^{176}$ P. Siddireddy, ${ }^{176}$ G. Smith, ${ }^{176}$ S. Taroni, ${ }^{176}$ M. Wayne, ${ }^{176}$ A. Wightman, ${ }^{176}$ M. Wolf, ${ }^{176}$ A. Woodard, ${ }^{176}$ J. Alimena, ${ }^{177}$ B. Bylsma, ${ }^{177}$ L. S. Durkin, ${ }^{177}$ S. Flowers, ${ }^{177}$ B. Francis, ${ }^{177}$

C. Hill, ${ }^{177}$ W. Ji, ${ }^{177}$ A. Lefeld, ${ }^{177}$ T. Y. Ling, ${ }^{177}$ B. L. Winer, ${ }^{177}$ S. Cooperstein, ${ }^{178}$ G. Dezoort, ${ }^{178}$ P. Elmer,${ }^{178}$ J. Hardenbrook, ${ }^{178}$ N. Haubrich, ${ }^{178}$ S. Higginbotham, ${ }^{178}$ A. Kalogeropoulos, ${ }^{178}$ S. Kwan, ${ }^{178}$ D. Lange, ${ }^{178}$ M. T. Lucchini, ${ }^{178}$ J. Luo, ${ }^{178}$ D. Marlow, ${ }^{178}$ K. Mei, ${ }^{178}$ I. Ojalvo, ${ }^{178}$ J. Olsen, ${ }^{178}$ C. Palmer, ${ }^{178}$ P. Piroué, ${ }^{178}$ J. Salfeld-Nebgen, ${ }^{178}$ D. Stickland, ${ }^{178}$ C. Tully, ${ }^{178}$ Z. Wang, ${ }^{178}$ S. Malik,${ }^{179}$ S. Norberg, ${ }^{179}$ A. Barker ${ }^{180}$ V. E. Barnes,${ }^{180}$ S. Das,${ }^{180}$ L. Gutay,${ }^{180}$ M. Jones, ${ }^{180}$ A. W. Jung, ${ }^{180}$ A. Khatiwada, ${ }^{180}$ B. Mahakud, ${ }^{180}$ D. H. Miller,${ }^{180}$ G. Negro, ${ }^{180}$ N. Neumeister, ${ }^{180}$ C. C. Peng, ${ }^{180}$

S. Piperov, ${ }^{180}$ H. Qiu, ${ }^{180}$ J. F. Schulte, ${ }^{180}$ J. Sun ${ }^{180}$ F. Wang, ${ }^{180}$ R. Xiao, ${ }^{180}$ W. Xie, ${ }^{180}$ T. Cheng, ${ }^{181}$ J. Dolen, ${ }^{181}$ N. Parashar, ${ }^{181}$ K. M. Ecklund, ${ }^{182}$ S. Freed, ${ }^{182}$ F. J. M. Geurts, ${ }^{182}$ M. Kilpatrick, ${ }^{182}$ Arun Kumar, ${ }^{182}$ W. Li, ${ }^{182}$ B. P. Padley, ${ }^{182}$ R. Redjimi, ${ }^{182}$ J. Roberts, ${ }^{182}$ J. Rorie, ${ }^{182}$ W. Shi, ${ }^{182}$ A. G. Stahl Leiton, ${ }^{182}$ Z. Tu, ${ }^{182}$ A. Zhang, ${ }^{182}$ A. Bodek, ${ }^{183}$ P. de Barbaro, ${ }^{183}$ R. Demina, ${ }^{183}$ J. L. Dulemba, ${ }^{183}$ C. Fallon, ${ }^{183}$ T. Ferbel, ${ }^{183}$ M. Galanti, ${ }^{183}$ A. Garcia-Bellido, ${ }^{183}$ J. Han, ${ }^{183}$ O. Hindrichs, ${ }^{183}$ A. Khukhunaishvili, ${ }^{183}$ E. Ranken, ${ }^{183}$ P. Tan, ${ }^{183}$ R. Taus, ${ }^{183}$ B. Chiarito, ${ }^{184}$ J. P. Chou, ${ }^{184}$ A. Gandrakota, ${ }^{184}$ Y. Gershtein, ${ }^{184}$ E. Halkiadakis, ${ }^{184}$ A. Hart, ${ }^{184}$ M. Heindl, ${ }^{184}$ E. Hughes, ${ }^{184}$ S. Kaplan, ${ }^{184}$ S. Kyriacou, ${ }^{184}$ I. Laflotte, ${ }^{184}$ 
A. Lath, ${ }^{184}$ R. Montalvo, ${ }^{184}$ K. Nash, ${ }^{184}$ M. Osherson, ${ }^{184}$ H. Saka, ${ }^{184}$ S. Salur, ${ }^{184}$ S. Schnetzer,${ }^{184}$ D. Sheffield, ${ }^{184}$ S. Somalwar, ${ }^{184}$ R. Stone, ${ }^{184}$ S. Thomas, ${ }^{184}$ P. Thomassen, ${ }^{184}$ H. Acharya ${ }^{185}$ A. G. Delannoy, ${ }^{185}$ J. Heideman, ${ }^{185}$ G. Riley, ${ }^{185}$ S. Spanier, ${ }^{185}$ O. Bouhali, ${ }^{186, v v v}$ A. Celik, ${ }^{186}$ M. Dalchenko, ${ }^{186}$ M. De Mattia, ${ }^{186}$ A. Delgado, ${ }^{186}$ S. Dildick, ${ }^{186}$ R. Eusebi, ${ }^{186}$ J. Gilmore, ${ }^{186}$ T. Huang, ${ }^{186}$ T. Kamon, ${ }^{186, \text { www }}$ S. Luo, ${ }^{186}$ D. Marley, ${ }^{186}$ R. Mueller, ${ }^{186}$ D. Overton, ${ }^{186}$ L. Perniè, ${ }^{186}$ D. Rathjens, ${ }^{186}$ A. Safonov, ${ }^{186}$ N. Akchurin, ${ }^{187}$ J. Damgov, ${ }^{187}$ F. De Guio, ${ }^{187}$ S. Kunori, ${ }^{187}$ K. Lamichhane, ${ }^{187}$ S. W. Lee, ${ }^{187}$ T. Mengke, ${ }^{187}$ S. Muthumuni, ${ }^{187}$ T. Peltola, ${ }^{187}$ S. Undleeb, ${ }^{187}$ I. Volobouev,${ }^{187}$ Z. Wang, ${ }^{187}$ A. Whitbeck, ${ }^{187}$ S. Greene, ${ }^{188}$ A. Gurrola, ${ }^{188}$ R. Janjam, ${ }^{188}$ W. Johns, ${ }^{188}$ C. Maguire, ${ }^{188}$ A. Melo, ${ }^{188}$ H. Ni ${ }^{188}$ K. Padeken, ${ }^{188}$ F. Romeo,${ }^{188}$ P. Sheldon, ${ }^{188}$ S. Tuo, ${ }^{188}$ J. Velkovska, ${ }^{188}$ M. Verweij, ${ }^{188}$ M. W. Arenton, ${ }^{189}$ P. Barria, ${ }^{189}$ B. Cox, ${ }^{189}$ G. Cummings, ${ }^{189}$ R. Hirosky, ${ }^{189}$ M. Joyce, ${ }^{189}$ A. Ledovskoy, ${ }^{189}$ C. Neu, ${ }^{189}$ B. Tannenwald,${ }^{189}$ Y. Wang, ${ }^{189}$ E. Wolfe,${ }^{189}$ F. Xia, ${ }^{189}$ R. Harr, ${ }^{190}$ P. E. Karchin, ${ }^{190}$ N. Poudyal,${ }^{190}$ J. Sturdy,${ }^{190}$ P. Thapa,${ }^{190}$ S. Zaleski,${ }^{190}$ J. Buchanan, ${ }^{191}$ C. Caillol,${ }^{191}$ D. Carlsmith, ${ }^{191}$ S. Dasu, ${ }^{191}$ I. De Bruyn, ${ }^{191}$ L. Dodd ${ }^{191}$ F. Fiori, ${ }^{191}$ C. Galloni, ${ }^{191}$ B. Gomber, ${ }^{191, \mathrm{xxx}}$ M. Herndon, ${ }^{191}$ A. Hervé, ${ }^{191}$ U. Hussain, ${ }^{191}$ P. Klabbers, ${ }^{191}$ A. Lanaro, ${ }^{191}$ A. Loeliger, ${ }^{191}$ K. Long, ${ }^{191}$ R. Loveless, ${ }^{191}$ J. Madhusudanan Sreekala,${ }^{191}$ T. Ruggles, ${ }^{191}$ A. Savin, ${ }^{191}$ V. Sharma, ${ }^{191}$ W. H. Smith, ${ }^{191}$ D. Teague, ${ }^{191}$ S. Trembath-reichert, ${ }^{191}$ and N. Woods ${ }^{191}$

\title{
(CMS Collaboration)
}

\author{
${ }^{1}$ Yerevan Physics Institute, Yerevan, Armenia \\ ${ }^{2}$ Institut für Hochenergiephysik, Wien, Austria \\ ${ }^{3}$ Institute for Nuclear Problems, Minsk, Belarus \\ ${ }^{4}$ Universiteit Antwerpen, Antwerpen, Belgium \\ ${ }^{5}$ Vrije Universiteit Brussel, Brussel, Belgium \\ ${ }^{6}$ Université Libre de Bruxelles, Bruxelles, Belgium \\ ${ }^{7}$ Ghent University, Ghent, Belgium \\ ${ }^{8}$ Université Catholique de Louvain, Louvain-la-Neuve, Belgium \\ ${ }^{9}$ Centro Brasileiro de Pesquisas Fisicas, Rio de Janeiro, Brazil \\ ${ }^{10}$ Universidade do Estado do Rio de Janeiro, Rio de Janeiro, Brazil \\ ${ }^{11 a}$ Universidade Estadual Paulista, São Paulo, Brazil \\ ${ }^{11 \mathrm{~b}}$ Universidade Federal do ABC, São Paulo, Brazil \\ ${ }^{12}$ Institute for Nuclear Research and Nuclear Energy, Bulgarian Academy of Sciences, Sofia, Bulgaria \\ ${ }^{13}$ University of Sofia, Sofia, Bulgaria \\ ${ }^{14}$ Beihang University, Beijing, China \\ ${ }^{15}$ Institute of High Energy Physics, Beijing, China \\ ${ }^{16}$ State Key Laboratory of Nuclear Physics and Technology, Peking University, Beijing, China \\ ${ }^{17}$ Tsinghua University, Beijing, China \\ ${ }^{18}$ Universidad de Los Andes, Bogota, Colombia \\ ${ }^{19}$ Universidad de Antioquia, Medellin, Colombia \\ ${ }^{20}$ University of Split, Faculty of Electrical Engineering, Mechanical Engineering and Naval Architecture, Split, Croatia \\ ${ }^{21}$ University of Split, Faculty of Science, Split, Croatia \\ ${ }^{22}$ Institute Rudjer Boskovic, Zagreb, Croatia \\ ${ }^{23}$ University of Cyprus, Nicosia, Cyprus \\ ${ }^{24}$ Charles University, Prague, Czech Republic \\ ${ }^{25}$ Escuela Politecnica Nacional, Quito, Ecuador \\ ${ }^{26}$ Universidad San Francisco de Quito, Quito, Ecuador \\ ${ }^{27}$ Academy of Scientific Research and Technology of the Arab Republic of Egypt, \\ Egyptian Network of High Energy Physics, Cairo, Egypt \\ ${ }^{28}$ National Institute of Chemical Physics and Biophysics, Tallinn, Estonia \\ ${ }^{29}$ Department of Physics, University of Helsinki, Helsinki, Finland \\ ${ }^{30}$ Helsinki Institute of Physics, Helsinki, Finland \\ ${ }^{31}$ Lappeenranta University of Technology, Lappeenranta, Finland \\ ${ }^{32} I R F U, C E A$, Université Paris-Saclay, Gif-sur-Yvette, France \\ ${ }^{33}$ Laboratoire Leprince-Ringuet, Ecole polytechnique, CNRS/IN2P3, Université Paris-Saclay, Palaiseau, France \\ ${ }^{34}$ Université de Strasbourg, CNRS, IPHC UMR 7178, Strasbourg, France \\ ${ }^{35}$ Centre de Calcul de l'Institut National de Physique Nucleaire et de Physique des Particules, CNRS/IN2P3, Villeurbanne, France \\ ${ }^{36}$ Université de Lyon, Université Claude Bernard Lyon 1, CNRS-IN2P3, Institut de Physique Nucléaire de Lyon, Villeurbanne, France \\ ${ }^{37}$ Georgian Technical University, Tbilisi, Georgia \\ ${ }^{38}$ Tbilisi State University, Tbilisi, Georgia \\ ${ }^{39}$ RWTH Aachen University, I. Physikalisches Institut, Aachen, Germany
}


${ }^{40}$ RWTH Aachen University, III. Physikalisches Institut A, Aachen, Germany

${ }^{41}$ RWTH Aachen University, III. Physikalisches Institut B, Aachen, Germany

${ }^{42}$ Deutsches Elektronen-Synchrotron, Hamburg, Germany

${ }^{43}$ University of Hamburg, Hamburg, Germany

${ }^{44}$ Karlsruher Institut fuer Technologie, Karlsruhe, Germany

${ }^{45}$ Institute of Nuclear and Particle Physics (INPP), NCSR Demokritos, Aghia Paraskevi, Greece

${ }^{46}$ National and Kapodistrian University of Athens, Athens, Greece

${ }^{47}$ National Technical University of Athens, Athens, Greece

${ }^{48}$ University of Ioánnina, Ioánnina, Greece

${ }^{49}$ MTA-ELTE Lendület CMS Particle and Nuclear Physics Group, Eötvös Loránd University, Budapest, Hungary

${ }^{50}$ Wigner Research Centre for Physics, Budapest, Hungary

${ }^{51}$ Institute of Nuclear Research ATOMKI, Debrecen, Hungary

${ }^{52}$ Institute of Physics, University of Debrecen, Debrecen, Hungary

${ }^{53}$ Eszterhazy Karoly University, Karoly Robert Campus, Gyongyos, Hungary

${ }^{54}$ Indian Institute of Science (IISc), Bangalore, India

${ }^{55}$ National Institute of Science Education and Research, HBNI, Bhubaneswar, India

${ }^{56}$ Panjab University, Chandigarh, India

${ }^{57}$ University of Delhi, Delhi, India

${ }^{58}$ Saha Institute of Nuclear Physics, HBNI, Kolkata, India

${ }^{59}$ Indian Institute of Technology Madras, Madras, India

${ }^{60}$ Bhabha Atomic Research Centre, Mumbai, India

${ }^{61}$ Tata Institute of Fundamental Research-A, Mumbai, India

${ }^{62}$ Tata Institute of Fundamental Research-B, Mumbai, India

${ }^{63}$ Indian Institute of Science Education and Research (IISER), Pune, India

${ }^{64}$ Institute for Research in Fundamental Sciences (IPM), Tehran, Iran

${ }^{65}$ University College Dublin, Dublin, Ireland

${ }^{66 a}$ INFN Sezione di Bari, Bari, Italy

${ }^{66 \mathrm{~b}}$ Università di Bari, Bari, Italy

${ }^{66 \mathrm{c}}$ Politecnico di Bari, Bari, Italy

${ }^{67 \mathrm{a} I N F N}$ Sezione di Bologna, Bologna, Italy

${ }^{67 \mathrm{~b}}$ Università di Bologna, Bologna, Italy

${ }^{68 \mathrm{a}}$ INFN Sezione di Catania, Catania, Italy

${ }^{68 \mathrm{~b}}$ Università di Catania, Catania, Italy

${ }^{69 \mathrm{a}}$ INFN Sezione di Firenze, Firenze, Italy

${ }^{69 \mathrm{~b}}$ Università di Firenze, Firenze, Italy

${ }^{70}$ INFN Laboratori Nazionali di Frascati, Frascati, Italy

${ }^{71 a}$ INFN Sezione di Genova, Genova, Italy

${ }^{71 \mathrm{~b}}$ Università di Genova, Genova, Italy

${ }^{72 a}$ INFN Sezione di Milano-Bicocca, Milano, Italy

${ }^{72 b}$ Università di Milano-Bicocca, Milano, Italy

${ }^{73 a}$ INFN Sezione di Napoli, Napoli, Italy

${ }^{73 b}$ Università di Napoli 'Federico II', Napoli, Italy

${ }^{73 \mathrm{c}}$ Università della Basilicata, Potenza, Italy

${ }^{73 \mathrm{~d}}$ Università G. Marconi, Roma, Italy

${ }^{74 a}$ INFN Sezione di Padova, Padova, Italy

${ }^{74 \mathrm{~b}}$ Università di Padova, Padova, Italy

${ }^{74 \mathrm{c}}$ Università di Trento, Trento, Italy

${ }^{75 a}$ INFN Sezione di Pavia, Pavia, Italy

${ }^{75 b}$ Università di Pavia, Pavia, Italy

${ }^{76 a}$ INFN Sezione di Perugia, Perugia, Italy

${ }^{76 \mathrm{~b}}$ Università di Perugia, Perugia, Italy

${ }^{77}$ INFN Sezione di Pisa, Pisa, Italy

${ }^{77 b}$ Università di Pisa, Pisa, Italy

${ }^{77 \mathrm{c}}$ Scuola Normale Superiore di Pisa, Pisa, Italy

${ }^{78 a}$ INFN Sezione di Roma, Rome, Italy

${ }^{78 \mathrm{~b}}$ Sapienza Università di Roma, Rome, Italy

${ }^{79 a}$ INFN Sezione di Torino, Torino, Italy

${ }^{79 b}$ Università di Torino, Torino, Italy

${ }^{79 c}$ Università del Piemonte Orientale, Novara, Italy

${ }^{80 \mathrm{a}}$ INFN Sezione di Trieste, Trieste, Italy 


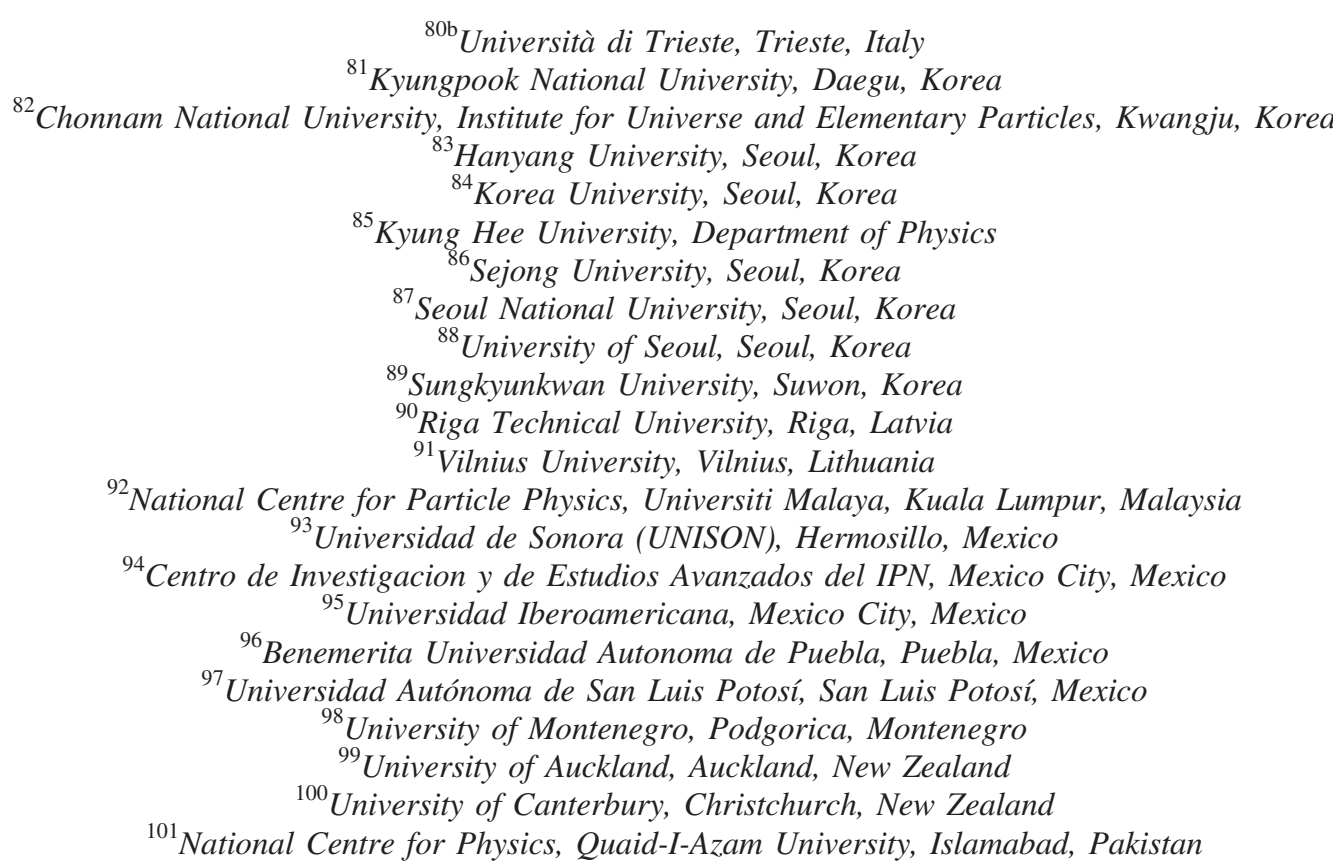

${ }^{102}$ AGH University of Science and Technology Faculty of Computer Science, Electronics and Telecommunications, Krakow, Poland

${ }^{103}$ National Centre for Nuclear Research, Swierk, Poland

${ }^{104}$ Institute of Experimental Physics, Faculty of Physics, University of Warsaw, Warsaw, Poland

${ }^{105}$ Laboratório de Instrumentação e Física Experimental de Partículas, Lisboa, Portugal

${ }^{106}$ Joint Institute for Nuclear Research, Dubna, Russia

${ }^{107}$ Petersburg Nuclear Physics Institute, Gatchina (St. Petersburg), Russia

${ }^{108}$ Institute for Nuclear Research, Moscow, Russia

${ }^{109}$ Institute for Theoretical and Experimental Physics named by A.I. Alikhanov of NRC 'Kurchatov Institute', Moscow, Russia

${ }^{110}$ Moscow Institute of Physics and Technology, Moscow, Russia

${ }^{111}$ National Research Nuclear University 'Moscow Engineering Physics Institute' (MEPhI), Moscow, Russia

${ }^{112}$ P.N. Lebedev Physical Institute, Moscow, Russia

${ }^{113}$ Skobeltsyn Institute of Nuclear Physics, Lomonosov Moscow State University, Moscow, Russia

${ }^{114}$ Novosibirsk State University (NSU), Novosibirsk, Russia

${ }^{115}$ Institute for High Energy Physics of National Research Centre 'Kurchatov Institute', Protvino, Russia

${ }^{116}$ National Research Tomsk Polytechnic University, Tomsk, Russia

${ }^{117}$ Tomsk State University, Tomsk, Russia

${ }^{118}$ University of Belgrade: Faculty of Physics and VINCA Institute of Nuclear Sciences, Belgrade, Serbia

${ }^{119}$ Centro de Investigaciones Energéticas Medioambientales y Tecnológicas (CIEMAT), Madrid, Spain

${ }^{120}$ Universidad Autónoma de Madrid, Madrid, Spain

${ }^{121}$ Universidad de Oviedo, Instituto Universitario de Ciencias y Tecnologías Espaciales de Asturias (ICTEA)

${ }^{122}$ Instituto de Física de Cantabria (IFCA), CSIC-Universidad de Cantabria, Santander, Spain

${ }^{123}$ University of Colombo, Colombo, Sri Lanka

${ }^{124}$ University of Ruhuna, Department of Physics, Matara, Sri Lanka

${ }^{125}$ CERN, European Organization for Nuclear Research, Geneva, Switzerland

${ }^{126}$ Paul Scherrer Institut, Villigen, Switzerland

${ }^{127}$ ETH Zurich-Institute for Particle Physics and Astrophysics (IPA), Zurich, Switzerland

${ }^{128}$ Universität Zürich, Zurich, Switzerland

${ }^{129}$ National Central University, Chung-Li, Taiwan

${ }^{130}$ National Taiwan University (NTU), Taipei, Taiwan

${ }^{131}$ Chulalongkorn University, Faculty of Science, Department of Physics, Bangkok, Thailand

${ }^{132}$ Çukurova University, Physics Department, Science and Art Faculty, Adana, Turkey

${ }^{133}$ Middle East Technical University, Physics Department, Ankara, Turkey

${ }^{134}$ Bogazici University, Istanbul, Turkey

${ }^{135}$ Istanbul Technical University, Istanbul, Turkey

${ }^{136}$ Istanbul University, Istanbul, Turkey

${ }^{137}$ Institute for Scintillation Materials of National Academy of Science of Ukraine, Kharkov, Ukraine

${ }^{138}$ National Scientific Center, Kharkov Institute of Physics and Technology, Kharkov, Ukraine

${ }^{139}$ University of Bristol, Bristol, United Kingdom 
${ }^{140}$ Rutherford Appleton Laboratory, Didcot, United Kingdom

${ }^{141}$ Imperial College, London, United Kingdom

${ }^{142}$ Brunel University, Uxbridge, United Kingdom

${ }^{143}$ Baylor University, Waco, Texas, USA

${ }^{144}$ Catholic University of America, Washington, DC, USA

${ }^{145}$ The University of Alabama, Tuscaloosa, Alabama, USA

${ }^{146}$ Boston University, Boston, Massachusetts, USA

${ }^{147}$ Brown University, Providence, Rhode Island, USA

${ }^{148}$ University of California at Davis, Davis, California, USA

${ }^{149}$ University of California at Los Angeles, California, USA

${ }^{150}$ University of California at Riverside, Riverside, California, USA

${ }^{151}$ University of California at San Diego, La Jolla, California, USA

${ }^{152}$ University of California at Santa Barbara, Santa Barbara-Department of Physics, Santa Barbara, California, USA

${ }^{153}$ California Institute of Technology, Pasadena, California, USA

${ }^{154}$ Carnegie Mellon University, Pittsburgh, Pennsylvania, USA

${ }^{155}$ University of Colorado Boulder, Boulder, Colorado, USA

${ }^{156}$ Cornell University, Ithaca, New York, USA

${ }^{157}$ Fermi National Accelerator Laboratory, Batavia, Illinois, USA

${ }^{158}$ University of Florida, Gainesville, Florida, USA

${ }^{159}$ Florida International University, Miami, Florida, USA

${ }^{160}$ Florida State University, Tallahassee, Florida, USA

${ }^{161}$ Florida Institute of Technology, Melbourne, Florida, USA

${ }^{162}$ University of Illinois at Chicago (UIC), Chicago, Illinois, USA

${ }^{163}$ The University of Iowa, Iowa City, Iowa, USA

${ }^{164}$ Johns Hopkins University, Baltimore, Maryland, USA

${ }^{165}$ The University of Kansas, Lawrence, Kansas, USA

${ }^{166}$ Kansas State University, Manhattan, Kansas, USA

${ }^{167}$ Lawrence Livermore National Laboratory, Livermore, California, USA

${ }^{168}$ University of Maryland, College Park, Maryland, USA

${ }^{169}$ Massachusetts Institute of Technology, Cambridge, Massachusetts, USA

${ }^{170}$ University of Minnesota, Minneapolis, Minnesota, USA

${ }^{171}$ University of Mississippi, Oxford, Mississippi, USA

${ }^{172}$ University of Nebraska-Lincoln, Lincoln, Nebraska, USA

${ }^{173}$ State University of New York at Buffalo, Buffalo, New York, USA

${ }^{174}$ Northeastern University, Boston, Massachusetts, USA

${ }^{175}$ Northwestern University, Evanston, Illinois, USA

${ }^{176}$ University of Notre Dame, Notre Dame, Indiana, USA

${ }^{177}$ The Ohio State University, Columbus, Ohio, USA

${ }^{178}$ Princeton University, Princeton, New Jersey, USA

${ }^{179}$ University of Puerto Rico, Mayaguez, Puerto Rico

${ }^{180}$ Purdue University, West Lafayette, Indiana, USA

${ }^{181}$ Purdue University Northwest, Hammond, Indiana, USA

${ }^{182}$ Rice University, Houston, Texas, USA

${ }^{183}$ University of Rochester, Rochester, New York, USA

${ }^{184}$ Rutgers, The State University of New Jersey, Piscataway, New Jersey, USA

${ }^{185}$ University of Tennessee, Knoxville, Tennessee, USA

${ }^{186}$ Texas A\&M University, College Station, Texas, USA

${ }^{187}$ Texas Tech University, Lubbock, Texas, USA

${ }^{188}$ Vanderbilt University, Nashville, Tennessee, USA

${ }^{189}$ University of Virginia, Charlottesville, Virginia, USA

${ }^{190}$ Wayne State University, Detroit, Michigan, USA

${ }^{191}$ University of Wisconsin-Madison, Madison, Wisconsin, USA

${ }^{\mathrm{a}}$ Deceased.

${ }^{\mathrm{b}}$ Also at Vienna University of Technology, Vienna, Austria.

${ }^{c}$ Also at IRFU, CEA, Université Paris-Saclay, Gif-sur-Yvette, France.

${ }^{\mathrm{d}}$ Also at Universidade Estadual de Campinas, Campinas, Brazil.

${ }^{\mathrm{e}}$ Also at Federal University of Rio Grande do Sul, Porto Alegre, Brazil.

${ }^{\mathrm{f}}$ Also at UFMS: Universidade Federal de Mato Grosso do Sul, Mato Grosso do Sul, Brazil.

${ }^{\mathrm{g}}$ Also at Universidade Federal de Pelotas, Pelotas, Brazil. 
${ }^{\mathrm{h}}$ Also at Université Libre de Bruxelles, Bruxelles, Belgium.

${ }^{\mathrm{i}}$ Also at University of Chinese Academy of Sciences, Bejing, China.

${ }^{j}$ Also at Institute for Theoretical and Experimental Physics named by A.I. Alikhanov of NRC 'Kurchatov Institute', Moscow, Russia.

${ }^{\mathrm{k}}$ Also at Joint Institute for Nuclear Research, Dubna, Russia.

${ }^{1}$ Also at Ain Shams University, Cairo, Egypt.

${ }^{\mathrm{m}}$ Also at Zewail City of Science and Technology, Zewail, Egypt.

${ }^{\mathrm{n}}$ Also at Purdue University, West Lafayette, Indiana, USA.

${ }^{\circ}$ Also at Université de Haute Alsace, Mulhouse, France.

${ }^{\mathrm{p}}$ Also at Erzincan Binali Yildirim University, Erzincan, Turkey.

${ }^{q}$ Also at CERN, European Organization for Nuclear Research, Geneva, Switzerland.

${ }^{\mathrm{r}}$ Also at RWTH Aachen University, III. Physikalisches Institut A, Aachen, Germany.

${ }^{\mathrm{s}}$ Also at University of Hamburg, Hamburg, Germany.

${ }^{t}$ Also at Brandenburg University of Technology, Cottbus, Germany.

"Also at Institute of Physics, University of Debrecen, Debrecen, Hungary.

${ }^{v}$ Also at Institute of Nuclear Research ATOMKI, Debrecen, Hungary.

${ }^{\text {w} A l s o ~ a t ~ M T A-E L T E ~ L e n d u ̈ l e t ~ C M S ~ P a r t i c l e ~ a n d ~ N u c l e a r ~ P h y s i c s ~ G r o u p, ~ E o ̈ t v o ̈ s ~ L o r a ́ n d ~ U n i v e r s i t y, ~ B u d a p e s t, ~ H u n g a r y . ~}$

${ }^{\mathrm{x}}$ Also at IIT Bhubaneswar, Bhubaneswar, India.

${ }^{\mathrm{y}}$ Also at Institute of Physics, Bhubaneswar, India.

${ }^{z}$ Also at Shoolini University, Solan, India.

${ }^{\text {aa }}$ Also at University of Visva-Bharati, Santiniketan, India.

${ }^{\mathrm{bb}}$ Also at Isfahan University of Technology, Isfahan, Iran.

${ }^{c c}$ Also at Italian National Agency for New Technologies, Energy and Sustainable Economic Development, Bologna, Italy.

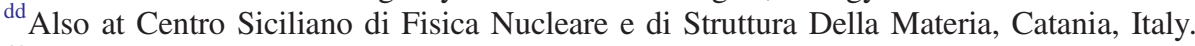

${ }^{e e}$ Also at Scuola Normale e Sezione dell'INFN, Pisa, Italy.

${ }^{\mathrm{ff}}$ Also at Riga Technical University, Riga, Latvia.

${ }^{g g}$ Also at Malaysian Nuclear Agency, MOSTI, Kajang, Malaysia.

${ }^{\text {hh }}$ Also at Consejo Nacional de Ciencia y Tecnología, Mexico City, Mexico.

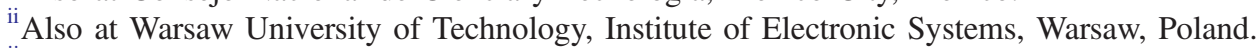

${ }^{\mathrm{jj}}$ Also at Institute for Nuclear Research, Moscow, Russia.

${ }^{k k}$ Also at National Research Nuclear University 'Moscow Engineering Physics Institute' (MEPhI), Moscow, Russia.

${ }^{11}$ Also at Institute of Nuclear Physics of the Uzbekistan Academy of Sciences, Tashkent, Uzbekistan.

${ }^{\mathrm{mm}}$ Also at St. Petersburg State Polytechnical University, St. Petersburg, Russia.

${ }^{\mathrm{nn}}$ Also at University of Florida, Gainesville, Florida, USA.

${ }^{\circ}$ Also at Imperial College, London, United Kingdom.

${ }^{\mathrm{pp}}$ Also at P.N. Lebedev Physical Institute, Moscow, Russia.

${ }^{\mathrm{qq}}$ Also at California Institute of Technology, Pasadena, California, USA.

${ }^{\text {rr }}$ Also at Budker Institute of Nuclear Physics, Novosibirsk, Russia.

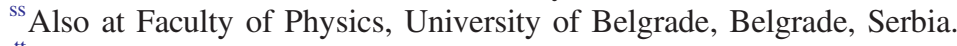

"Also at Università degli Studi di Siena, Siena, Italy.

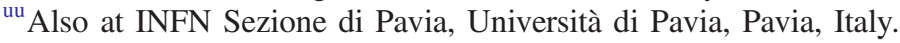

${ }^{\mathrm{vv}}$ Also at National and Kapodistrian University of Athens, Athens, Greece.

${ }^{\text {ww }}$ Also at Universität Zürich, Zurich, Switzerland.

${ }^{x x}$ Also at Stefan Meyer Institute for Subatomic Physics, Vienna, Austria.

${ }^{\text {yy }}$ Also at Adiyaman University, Adiyaman, Turkey.

${ }^{\mathrm{zz}}$ Also at Sirnak University, Sirnak, Turkey.

${ }^{\text {aaa }}$ Also at Beykent University, Istanbul, Turkey.

${ }^{b b b}$ Also at Istanbul Aydin University, Istanbul, Turkey.

${ }^{c c c}$ Also at Mersin University, Mersin, Turkey.

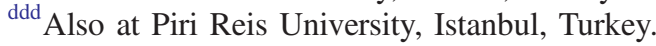

${ }^{e e e}$ Also at Gaziosmanpasa University, Tokat, Turkey.

${ }^{\text {fff }}$ Also at Ozyegin University, Istanbul, Turkey.

${ }^{\text {ggg }}$ Also at Izmir Institute of Technology, Izmir, Turkey.

hhh Also at Marmara University, Istanbul, Turkey.

iii Also at Kafkas University, Kars, Turkey.

${ }^{\mathrm{jij}}$ Also at Istanbul Bilgi University, Istanbul, Turkey.

${ }^{k k k}$ Also at Hacettepe University, Ankara, Turkey.

${ }^{111}$ Also at School of Physics and Astronomy, University of Southampton, Southampton, United Kingdom.

${ }^{\mathrm{mmm}}$ Also at IPPP Durham University, Durham, England.

${ }^{n n n}$ Also at Monash University, Faculty of Science, Clayton, Australia.

${ }^{000}$ Also at Bethel University, St. Paul, Minneapolis, USA. 


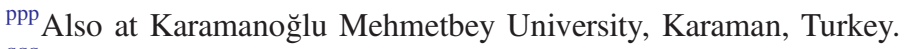

${ }^{\mathrm{qqq}}$ Also at Vilnius University, Vilnius, Lithuania.

${ }^{\mathrm{rrr}}$ Also at Bingol University, Bingol, Turkey.

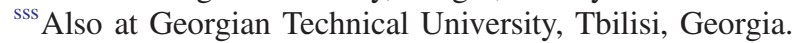

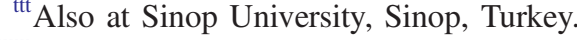

${ }^{u u u}$ Also at Mimar Sinan University, Istanbul, Istanbul, Turkey.

${ }^{\mathrm{vvv}}$ Also at Texas A\&M University at Qatar, Doha, Qatar.

${ }^{w w w}$ Also at Kyungpook National University, Daegu, Korea.

${ }^{\mathrm{xxx}}$ Also at University of Hyderabad, Hyderabad, India. 